asHOKa

UNIVERSITY

ASHOKA UNIVERSITY ECONOMICS

DISCUSSION PAPER NO. 38

\title{
Evolutionary Implementation in Aggregative Games
}

September 2020

Ratul Lahkar, Ashoka University

Saptarshi Mukherjee, Indian Institute of Technology, Delhi 


\title{
Evolutionary Implementation in Aggregative Games
}

\author{
Ratul Lahkar* Saptarshi Mukherjee S $^{\dagger}$
}

July 20, 2020

\begin{abstract}
Due to externalities, the equilibrium behavior in aggregative games is not efficient in the sense of maximizing aggregate payoff. We characterize conditions such that efficiency can be globally implemented in such games under evolutionary dynamics. If payoffs satisfy certain important concavity conditions, then the aggregate payoff function of these games has a unique maximizer. Once the planner imposes a transfer equal to the externality generated by agents, we obtain a new externality adjusted game. This is a potential game with the aggregate payoff function of the original game being its potential function. Evolutionary dynamics converge globally to the maximizer of this potential function, thereby implementing efficiency in the original game. Our earlier paper on public goods (Lahkar and Mukherjee [16]) emerges as an example of the present general analysis. Two new applications are public bads and the tragedy of the commons.
\end{abstract}

Keywords: Aggregative Games; Externalities; Potential Games; Implementation.

JEL classification: C72; C73; D62; D82.

*(corresponding author) Department of Economics, Ashoka University, Rajiv Gandhi Education City, Sonipat, Haryana 131029, India, email: ratul.lahkar@ashoka.edu.in.

${ }^{\dagger}$ Department of Humanities and Social Sciences, Indian Institute of Technology Delhi, Hauz Khas, New Delhi, India, email: saptarshi.isi@gmail.com 


\section{Introduction}

Implementing efficient outcomes has been a topic of abiding interest in economics and is the key objective in the mechanism design literature. The approach in much of this literature is that a planner designs a mechanism upon which agents instantaneously coordinate on the efficient outcome desired by the planner. The most well known class of mechanisms is the Vickrey-Clarke-Groves (VCG) direct mechanism that renders truthful revelation of type the dominant strategy for each agent (Vickrey [36], Clarke [6], Groves [9]). Several authors have, however, noted practical problems with this approach to implementation, particularly when the number of agents involved is large; for example, collecting information about types from a large number of agents and computing assignments based upon reported types (Sandholm [33]), concern about revealing confidential information about types and the possibility of cheating by the bid taker and competing bidders (Rothkopf et al. [29], Rothkopf [30]). In view of such problems, an alternative approach to implementation has emerged which views the problem from an evolutionary perspective in which optimal strategies in a mechanism design problem emerges gradually rather than instantaneously (Sandholm [32, 33, 34], Phelps et al. [28], Lahkar and Mukherjee [16]).

In this paper, we adopt this evolutionary approach to implementation in a particular class of games called large population aggregative games. These are games played by a large population (a continuum) of agents in which the payoffs depend upon an agent's own strategy and the aggregate strategy level in the society. ${ }^{1}$ Aggregative games are of interest because of their analytical tractability and the fact that important economic applications can be modeled as such games. One such application is the public goods problem considered in our earlier paper (Lahkar and Mukherjee [16]). In this paper, we generalize our earlier approach to a wider class of aggregative games and analyze two new applications-public bads and the tragedy of the commons. Due to the dependence of payoffs on aggregate strategy, aggregative games are beset by the problem of externalities. This typically causes a distinction between the Nash equilibrium and the efficient state, where the efficient state is the state that maximizes aggregate payoff in the society. For example, in the large population public goods game considered in Lahkar and Mukherjee [16], externalities are positive because higher contribution by an agent enhances the welfare of others. Hence, the efficient state requires significant positive contribution by all agents whereas the Nash equilibrium entails the minimum possible contribution.

The solution to this problem is to implement a variable externality pricing scheme (Sandholm [32]) to the original game. Under this scheme, a transfer equal to the externality imposed by an agent is made to the agent, with the externality being calculated with respect to the current social state. Sandholm [32] shows that the resulting externality adjusted game is a potential game (Monderer and Shapley [22], Sandholm [31]) with the aggregate payoff function of the original game

\footnotetext{
${ }^{1}$ The large population characteristic of these games allow us to rigorously apply methods of evolutionary game theory to establish convergence to the efficient state. This characteristic is, of course, a formalization of a situation in which the number of agents are finite but large, which is precisely the situation when the classical approach to mechanism design may encounter practical problems alluded to earlier.
} 
as its potential function. This is important because all well-known dynamics in evolutionary game theory converge to a maximizer of the potential function in a potential game (Sandholm [31]). Hence, if the aggregate payoff function of the original aggregative game has a unique maximizer, and we identify conditions such that it does, then evolutionary dynamics in the externality adjusted game will converge globally to its maximizer, which is the efficient state.

This is the key insight behind evolutionary implementation as developed by Sandholm [32]. ${ }^{2}$ Lahkar and Mukherjee's [16] analysis of the public goods problem extends this idea in two important ways. First, they allowed for a more general payoff function which need not be decomposable into a common and an idiosyncratic part, as was the case with Sandholm's models. Second, they allowed the strategy set in their model to be continuous. While this approach raises certain measure theoretic complications, it is useful in an aggregative game like the public goods model in allowing for a more exact and parsimonious characterization of the Nash equilibrium and the efficient state. ${ }^{3}$ We discuss both these features of aggregative games in further detail in our earlier paper. Using the potential game approach of Sandholm [32, 33], that paper establishes evolutionary implementation of the efficient state in the public goods under standard deterministic evolutionary dynamics. In this paper, we retain the same framework as Lahkar and Mukherjee [16] by considering an aggregative game with a continuous strategy set and multiple types of agents, each type characterized by a distinct payoff function. As in that paper, the continuous nature of the strategy set simplifies the characterization of efficiency greatly. We then make the following contributions beyond our earlier paper.

First, we generalize evolutionary implementation to a broader class of aggregative games that includes two new applications-public bads and the tragedy of the commons. In economics, these models are used to represent a wide variety of social problems. Our evolutionary analysis provides an important insight into how a large society may be nudged towards the optimal outcome in such situations. Second, we identify the broader class of aggregative games where global evolutionary implementation holds. These are games which satisfy two important concavity conditions-strict concavity of the payoff function of the game with respect to individual strategy (Assumption 3.1) and a weaker concavity condition of the aggregate strategy level with respect to the social state (Assumption 3.3). These conditions ensure that the aggregate payoff function of an aggregative game has a unique maximizer, to which evolutionary dynamics must converge globally in the externality adjusted game. ${ }^{4}$ Third, we generalize the method of characterizing the unique efficient state of any aggregative game that satisfies the aforementioned concavity conditions (Propositon

\footnotetext{
${ }^{2}$ Sandholm [32] considers implementation under deterministic dynamics in a congestion game. Sandholm [33] generalizes this model by adding idiosyncratic payoffs. Both were models of negative externalities. Sandholm [34] considers stochastic evolution which also allows for positive externalities.

${ }^{3}$ See Appendix A.2.1 in Lahkar and Mukherjee [16] for a more detailed discussion of the advantages of the continuous strategy approach over a finite strategy approach in aggregative games.

${ }^{4}$ We establish convergence under the same evolutionary dynamics we considered in our earlier paper. These are the replicator dynamic (Oechssler and Riedel [23], [24], Cheung [4]), the Brown-von Neumann-Nash (BNN) dynamic (Hofbauer et al. [12]), the pairwise comparison dynamic (Cheung [3]), the logit dynamic (Perkins and Leslie [26], Lahkar and Riedel [14]) and the best response dynamic for aggregative games with continuous strategy set (Lahkar and Mukherjee [16]).
} 
3.5). This method is computationally simple but depends crucially upon the aggregative nature of the game. Indeed, applications aside, this analytical tractability is a major reason why we focus on such games. Finally, by generalizing evolutionary implementation to aggregative games with continuous strategy sets, this paper also widens the applicability of the continuous strategy approach to evolutionary game theory that was pioneered by Oechssler and Riedel [23, 24].

The public goods model in our earlier paper was an example of positive externalities. The two other applications in the present paper are examples of negative externalities. Together, these three examples provide another general insight about aggregative games. It is that the important concavity conditions that ensure a unique maximizer of the aggregate payoff function are naturally satisfied in such games irrespective of the nature of externalities. This is of significance because we can then apply deterministic evolution to such games to globally implement efficiency. Convergence under deterministic dynamics is fairly rapid. On the other hand, in the type of applications considered by Sandholm ([32],[33],[34]), negative externalities do ensure concavity and, hence, a unique maximizer of the aggregate payoff function. But under positive externalities, the relevant payoff functions may be convex which implies that the aggregate payoff function has multiple local maximizers. In that case, global implementation of efficiency would have required the use of stochastic evolutionary methods (Sandholm [34]). Convergence under stochastic methods is, however, much slower than under deterministic methods. ${ }^{5}$

One question that may arise is why do we need the general analysis of this paper. Couldn't we have followed the specific approach of our earlier public goods model and analyzed the present applications by relying on the particular features of these models? For the public bads application, we could have. In fact, for this application, we could have derived our conclusions simply as corollaries to our earlier results in Lahkar and Mukherjee [16] by reversing the order structure on the strategy set. But the general analysis serves the role of highlighting the underlying similarities between these models despite their economic differences. Externalities are different in the two models as well as the origins of those externalities. ${ }^{6}$ Nevertheless, both models satisfy the important concavity conditions of this paper which drives evolutionary implementation. A straightforward application of the public goods approach to the tragedy of the commons is difficult. The tragedy of the commons is fundamentally different in that it models a common resource instead of a public resource. This is reflected in the payoff functions of this model where even the benefit part depends upon individual strategy whereas in the public goods model, benefits are independent of individual strategy. In particular, this makes characterization of Nash equilibrium more challenging in this model. $^{7}$ Yet, despite these conceptual and technical differences, our general analysis shows that there are fundamental similarities in the public resource and common resource models which drive evolutionary implementation. The analysis of the tragedy of the commons also shows that our

\footnotetext{
${ }^{5}$ Oyama et al. [25], for example, provide a discussion of the difference in the speed of convergence under the deterministic and stochastic approaches to evolutionary game theory.

${ }^{6}$ For the public goods model, the positive externalities originate in the public nature of benefits. For the public bads model, the negative externaltities originate in the public nature of costs.

${ }^{7}$ As we show in Section 5.2, solving for Nash equilibrium in the tragedy of the commons itself requires us to use the technique of potential games.
} 
methodology of evolutionary implementation in aggregative games is more widely applicable than just models of public resources.

The idea of a variable externality pricing scheme is closely related to the classical notion of Pigouvian pricing (Pigou [27]). Both schemes rely on imposing a transfer on agents that force them to internalize the externality they create. The key difference is that the Pigouvian price is calculated with respect to the externality at the efficient state, evolutionary implementation calculates the transfer with respect to the level of externality. Thus, while both implements the efficient solution, evolutionary implementation is informationally less demanding. The planner may not know the efficient state a priori which would make calculating the Pigouvian price difficult. In contrast, there are well established empirical methods to calculate current externality (Lin [20]). In our view, therefore, evolutionary implementation provides a more feasible way to combat externalities. We discuss this issue in further detail in Section 6.

The rest of the paper is as follows. In Section 2, we discuss the general model of population games with continuous strategy sets and define externalities in such games. We also define potential games and show that the externality adjusted game is a potential game. Section 3 applies these results to aggregative games and characterizes the efficient state in such game. Section 4 discusses evolutionary implementation. In Section 5, we apply our model to the public bads model and the tragedy of the commons. Section 6 discusses the difference between Pigouvian pricing and evolutionary implementation. Section 7 concludes.

\section{Population Games with Continuous Strategy Sets}

We consider a society consisting of a continuum of agents of mass 1 . The society is divided into a finite set of types or populations $\mathcal{P}=\{1,2, \cdots, n\}$. Agents of a particular type are characterized by a common payoff function. The mass of population $p$ is $m_{p} \in(0,1)$, with $\sum_{p \in \mathcal{P}} m_{p}=1$. Given our interpretation of each population as a type, the mass distribution $m=\left(m_{1}, \cdots, m_{n}\right)$ also constitutes the type distribution in the society.

In view of the applications we consider later in this paper, we assume every agent in the society has a common strategy set $\mathcal{S}=[\underline{x}, \bar{x}] \subset \mathbf{R}_{+}$, with $\underline{x} \geq 0$. The continuous-strategy framework of our model requires us to introduce certain measure-theoretic notations to describe population states. Let $\mathcal{B}$ be the Borel $\sigma$-algebra on $\mathcal{S}$. We denote by $\mathcal{M}(\mathcal{S})$ the space of finite signed measures on $(\mathcal{S}, \mathcal{B})$. The subset $\mathcal{M}_{m}^{+}(\mathcal{S}) \subset \mathcal{M}(\mathcal{S})$ is then the space of finite measures that impose a total mass of $m>0$ on $\mathcal{S}$. Thus, $\mathcal{M}_{1}^{+}(\mathcal{S})$ is the space of probability measures on $\mathcal{S}$. We also use the notation $\mathcal{M}$ to denote the cross product of $\mathcal{M}(\mathcal{S})$ with itself $n$-times. Thus, $\mathcal{M}=\prod_{p=1}^{n} \mathcal{M}(\mathcal{S})$. Further, we denote by $\mathfrak{M}_{b}(\mathcal{S} \times \mathcal{P})$ the space of bounded measurable functions on $\mathcal{S} \times \mathcal{P}$ with the supremum norm.

The set $\mathcal{M}_{m_{p}}^{+}(\mathcal{S}) \subset \mathcal{M}(\mathcal{S})$ then constitutes the set of states in population $p$, with $\mu_{p} \in \mathcal{M}_{m_{p}}^{+}(\mathcal{S})$ being such a population state. The interpretation of $\mu_{p}$ is that $\mu_{p}(A) \in\left[0, m_{p}\right]$ represents the mass of agents in population $p$ who play strategies in $A \subseteq \mathcal{S}$. Of particular interest to us are 
monomorphic population states, which are states in which every agent in a population $p$ plays the same strategy. We denote a monomorphic population state for $p \in \mathcal{P}$ in which every agent in that population plays $x_{p} \in \mathcal{S}$ as $m_{p} \delta_{x_{p}}{ }^{8}$ If a population state is not monomorphic, then we call it polymorphic.

To define a population game, we denote the set of states in the entire society as $\Delta=\prod_{p=1}^{n} \mathcal{M}_{m_{p}}^{+}(\mathcal{S})$. A social state is, therefore, $\mu=\left(\mu_{1}, \cdots, \mu_{n}\right) \in \Delta$, where $\mu_{p} \in \mathcal{M}_{m_{p}}^{+}(\mathcal{S})$. A population game is then a weakly continuous mapping

$$
F: \Delta \rightarrow \mathfrak{M}_{b}(\mathcal{S} \times \mathcal{P})
$$

such that $F_{x, p}(\mu)$ is the payoff of an agent in population $p$ who uses strategy $x \in \mathcal{S}$ at the social state $\mu$. We define the Nash equilibrium of $F$ as follows.

Definition 2.1 A Nash equilibrium of a multipopulation game $F$ as defined in (1) is a social state $\mu^{*}=\left(\mu_{1}^{*}, \mu_{2}^{*}, \cdots, \mu_{n}^{*}\right) \in \Delta$ such that for all $x \in \mathcal{S}$, all $p \in \mathcal{P}$, if $x$ lies in the support of $\mu_{p}^{*}$, then $F_{x, p}\left(\mu^{*}\right) \geq F_{y, p}\left(\mu^{*}\right)$, for all $y \in \mathcal{S}$.

Our analysis will require the notion of the aggregate payoff in $F$. For this purpose, we introduce the function $\bar{F}: \Delta \rightarrow \mathbf{R}$. We then define the aggregate payoff in the population game $F$ defined by (1) at a social state $\mu \in \Delta$ as

$$
\bar{F}(\mu)=\sum_{p \in \mathcal{P}} \int_{\mathcal{S}} F_{x, p}(\mu) \mu_{p}(d x) .
$$

This leads to the following definition of an efficient state in $F$.

Definition 2.2 An efficient state of a multipopulation game $F$ as defined in (1) is a social state $\mu^{* *}=\left(\mu_{1}^{* *}, \mu_{2}^{* *}, \cdots, \mu_{n}^{* *}\right) \in \Delta$ that maximizes the aggregate payoff $\bar{F}$ as defined in (2).

Much of the analysis in this paper will be based on the notion of externalities in $F$. In a large population game like $F$, the externality imposed by strategy $x$ users in population $p$ on strategy $y$ users in population $q$ is the marginal impact on the payoff of $y$-users in population $q$ when the mass of $x$-users in population $p$ increases (Sandholm ([31])). When we sum up over all strategies in all populations, we obtain the total externality imposed by strategy $x$ users in population $p$. Lahkar and Mukherjee [16], building upon the work of Cheung and Lahkar [5] in the context of single population games, provide a formal definition of externalities for a multipopulation game of the form (1) which has a continuous strategy set. We describe that definition as follows.

Calculating externalties for a population game $F$ with a continuous strategy set requires the notion of the Fréchet derivative. This is a generalization of the usual concept of the derivative to Banach spaces. ${ }^{9}$ To define the Fréchet derivative for a payoff function $F_{y, q}(\mu)$, we first extend the domain of $F_{y, q}$ from $\Delta$ to $\mathcal{M}$. Then, the Fréchet derivative of $F_{y, q}(\mu)$ in the direction $\zeta \in \mathcal{M}$,

\footnotetext{
${ }^{8}$ Here, $\delta_{x_{p}}$ is the Dirac distribution with probability 1 on $x_{p}$.

${ }^{9}$ See Definition A.1 in Appendix A.1 for the formal definition.
} 
denoted, $D F_{y, q}(\mu) \zeta$, represents the change in $F_{y, q}$ when $\mu$ changes in the direction $\zeta$. With this understanding of the Fréchet derivative, we define the total externality imposed by agents of type $p$ who play strategy $x$ at the social state $\mu$ as

$$
e_{x, p}(\mu)=\sum_{q \in \mathcal{P}} \int_{\mathcal{S}} D F_{y, q}(\mu) \mathcal{D}_{x, p} \mu_{q}(d y)
$$

where $D F_{y, q}(\mu)$ is the Fréchet derivative of the payoff function $F_{y, q}(\mu)$ and $\mathcal{D}_{x, p}=\zeta \in \mathcal{M}$ such that $\zeta_{p}=\delta_{x}$ and $\zeta_{k}=0$ for all $k \in \mathcal{P} \backslash p$.

Intuitively, the measure $\zeta=\mathcal{D}_{x, p}$ represents a situation where the only change in $\mu$ is an increase in the mass of agents using strategy $x$ in population $p$. Hence, $D F_{y, q}(\mu) \mathcal{D}_{x, p}$ in $(3)$ is the change in the payoff of strategy $y$-users in population $q$ when the mass of strategy $x$-users in population $p$ changes. Summing up over all strategies $y \in \mathcal{S}$ and all populations $p \in \mathcal{P}$ then gives us (3). Further details of applying the Fréchet derivative to arrive at this definition of total externalities are in Appendix A.1.1 in Lahkar and Mukherjee [16].

\subsection{Potential Games and the Externality Adjusted Game}

Using (3), we define the externality adjusted game $\hat{F}$ as follows.

$$
\begin{aligned}
\hat{F}_{x, p}(\mu) & =F_{x, p}(\mu)+e_{x, p}(\mu) \\
& =F_{x, p}(\mu)+\sum_{q \in \mathcal{P}} \int_{\mathcal{S}} D F_{y, q}(\mu) \mathcal{D}_{x, p} \mu_{q}(d y) .
\end{aligned}
$$

We wish to show that $\hat{F}$ is a potential game with potential function $\bar{F}$ defined in (2). The definition depends upon the notion of a gradient of a Fréchet differentiable function. Consider $f: \mathcal{M} \rightarrow \mathbf{R}$ that is Fréchet differentiable. Let $D f(\mu) \zeta$ be the Fréchet derivative of $f$ at $\mu \in \mathcal{M}$ in the direction $\zeta=\left(\zeta_{1}, \cdots, \zeta_{n}\right) \in \mathcal{M}$. Suppose there exists an element $\nabla f(\mu): \mathcal{S} \times \mathcal{P} \rightarrow \mathfrak{M}_{b}(\mathcal{S} \times \mathcal{P})$ such that

$$
D f(\mu) \zeta=\sum_{p \in \mathcal{P}} \int_{\mathcal{S}} \nabla f(\mu)(x, p) \zeta_{p}(d x)=\langle\nabla f(\mu), \zeta\rangle, \text { for all } \zeta=\left(\zeta_{1}, \cdots, \zeta_{n}\right) \in \mathcal{M}
$$

where we use the "inner product" notation $\langle\cdot, \cdot\rangle: \mathfrak{M}_{b}(\mathcal{S} \times \mathcal{P}) \times \mathfrak{M} \rightarrow \mathbf{R}$ to denote $\langle g, \nu\rangle=$ $\sum_{p \in \mathcal{P}} \int_{\mathcal{S}} g(x, p) \nu_{p}(d x)$, for $g \in \mathfrak{M}_{b}(\mathcal{S} \times \mathcal{P})$ and $\nu \in \mathfrak{M}$. This leads to the following definition of a multipopulation potential game with a continuous strategy set (Lahkar and Mukherjee [16]).

Definition 2.3 A population game $F: \Delta \rightarrow \mathfrak{M}_{b}(\mathcal{S} \times \mathcal{P})$ as defined in (1) is a potential game if there exists a Fréchet differentiable (with respect to the variational norm) function $f: \mathcal{M} \rightarrow \mathbf{R}$ such that

$$
\nabla f(\mu)=F(\mu) \text { for all } \mu=\left(\mu_{1}, \cdots, \mu_{n}\right) \in \Delta .
$$

The function $f$ is called the potential function of the game $F$. 
Thus, for a multipopulation game $F$ to be a potential game with potential function $f$, we need to show that for every $\mu \in \Delta$ and every $(x, p) \in \mathcal{S}, \nabla f(\mu)(x, p)=F_{x, p}(\mu)$. We apply Definition 2.3 to arrive at our desired result that $\hat{F}$ is a potential game with potential function $\bar{F}$. This result is a generalization of the result in Example 3 of Cheung [3] to the multipopulation case. The proof is in Appendix A.1.

Proposition 2.4 The population game $\hat{F}$ defined by (4) is a potential game with potential function $\bar{F}$ defined by (2).

\section{Aggregative Games}

Our interest is in one particular class of population games, which are aggregative games. These are games in which the payoff to an agent depends upon the agent's own strategy and the aggregate strategy level in the society (Corchón [7]). The aggregate strategy level at the social state $\mu=$ $\left(\mu_{1}, \cdots, \mu_{n}\right)$ is

$$
A(\mu)=\sum_{p \in \mathcal{P}} \int_{\mathcal{S}} x \mu_{p}(d x)=\int_{\mathcal{S}} x \sum_{p \in \mathcal{P}} \mu_{p}(d x) .
$$

Note that $A(\mu) \in[\underline{x}, \bar{x}]$. In our subsequent discussion, we will frequently use $\alpha$ to denote a particular value of $A(\mu)$.

Formally, we say that the population game $F$ as defined in (1) is an aggregative game if the payoff to an agent in population $p$ playing $x \in \mathcal{S}$ is

$$
F_{x, p}(\mu)=\beta_{p}(x, A(\mu))
$$

where $A(\mu)$ is the aggregate strategy level as defined in (6). Apart from standard continuity and differentiablity assumptions, we also assume that $\beta_{p}$ satisfies the following property.

Assumption 3.1 Consider the aggregative game $F$ as defined in (7). We assume that for every $\alpha=A(\mu), \beta_{p}(x, \alpha)$ is strictly concave in $x$.

As an example of an aggregative game of the form (7), we present the public goods game analyzed in Lahkar and Mukherjee [16].

Example 3.2 Consider an agent in population $p$ using strategy $x \in \mathcal{S}$. Let $\beta_{p}(x, \alpha)=v_{p}(\alpha)-c_{p}(x)$ with $c_{p}$ being strictly convex for all $p \in \mathcal{P}$. Then, $F_{x, p}(\mu)=\beta_{p}(x, p)$ constitutes a public goods game with multiple types. In this game, $v_{p}$ denotes the benefit to the agent while $c_{p}$ describes the cost. The public goods character of this model arises because the benefit depends entirely upon the aggregate strategy level while the cost is entirely a function of individual strategy. Note that the strict convexity of $c_{p}$ implies that Assumption 3.1 is satisfied. 


\subsection{Efficient State}

The aggregate payoff in the aggregative game (7) is

$$
\bar{F}(\mu)=\sum_{p \in \mathcal{P}} \int_{\mathcal{S}} \beta_{p}(x, A(\mu)) \mu_{p}(d x) .
$$

Thus, an efficient state in the aggregative game is a state $\mu^{* *}$ that maximizes (8). We make the following important assumption about the aggregate payoff.

Assumption 3.3 Consider the aggregate payoff function $\bar{F}$ defined in (8) for the aggregative game (7). Let $\mu, \nu \in \Delta$. Suppose $A(\mu) \neq A(\nu)$. Then, for all $\lambda \in(0,1), \bar{F}(\lambda \mu+(1-\lambda) \nu)>\lambda \bar{F}(\mu)+$ $(1-\lambda) \bar{F}(\nu)$. Thus, if $A(\mu) \neq A(\nu)$, then

$$
\begin{aligned}
\sum_{p \in \mathcal{P}} \int_{\mathcal{S}} \beta_{p}(x, A(\lambda \mu+(1-\lambda) \nu))\left(\lambda \mu_{p}+(1-\lambda) \nu_{p}\right)> & \lambda \sum_{p \in \mathcal{P}} \int_{\mathcal{S}} \beta_{p}(x, A(\mu)) \mu_{p}(d x) \\
& +(1-\lambda) \sum_{p \in \mathcal{P}} \int_{\mathcal{S}} \beta_{p}(x, A(\nu)) \nu_{p}(d x) .
\end{aligned}
$$

The importance of Assumption 3.3 will lie in enabling us to characterize the maximizer of $\bar{F}$ defined by (8). Had $\bar{F}$ been strictly concave, it would have followed immediately that it has a unique maximizer. But by its definition in (8), $\bar{F}$ cannot be strictly concave. For $\mu, \nu$ such that $A(\mu)=A(\nu)$, the two sides of (9) must hold with equality. Assumption 3.3, though, does imply that $\bar{F}$ is as strictly concave as it can be. As long as $A(\mu) \neq A(\nu)$, the condition for strict concavity, which is (9), holds. This does suggest that $\bar{F}$ should have a unique maximizer. Once we introduce Assumption 3.1, this turns out to be true. Moreover, that maximizer is in monomorphic population states. We establish these conclusions in the following lemma. The proof is in Appendix A.2.

Lemma 3.4 Suppose Assumptions 3.1 and 3.3 hold. Then, the aggregate payoff function $\bar{F}$ defined by (8) for the aggregative game (7) is concave but not strictly concave. Further, if $\mu^{* *} \in \Delta$ maximizes $\bar{F}$, then, for every $p \in \mathcal{P}, \mu_{p}^{* *}$ is a monomorphic state. Hence, $\bar{F}$ defined by (8) has a unique maximizer.

We, therefore, conclude from Lemma 3.4 that the aggregative game $F$ defined by (7) has a unique efficient state. Characterizing this efficient state by maximizing $\bar{F}$ directly is, however, difficult as $\bar{F}$ is defined on an abstract measure space. Instead, we introduce the function $\bar{G}: \prod_{p=1}^{n}[\underline{x}, \bar{x}] \rightarrow \mathbf{R}$ such that

$$
\bar{G}\left(\alpha_{1}, \alpha_{2}, \cdots, \alpha_{n}\right)=\sum_{p \in \mathcal{P}} m_{p} \beta_{p}\left(\alpha_{p}, \sum_{q \in \mathcal{P}} m_{q} \alpha_{q}\right) .
$$

It is evident from (8) and (10) that if $\mu=\left(m_{1} \delta_{\alpha_{1}}, \cdots, m_{n} \delta_{\alpha_{n}}\right)$, then $\bar{F}(\mu)=\bar{G}\left(\alpha_{1}, \cdots, \alpha_{n}\right)$. Since, by Lemma 3.4, the maximizer of $\bar{F}$ must be in monomorphic population states, it suffices to maximize $\bar{G}$ in order to characterize the efficient state of the aggregative game $F$. This is useful 
because maximizing $\bar{G}$ is a simple exercise. We state the result formally in the following proposition. The proof is in Appendix A.2.

Proposition 3.5 Consider the aggregative game $F$ defined in (7). Let Assumptions 3.1 and 3.3 hold. Then, $F$ has a unique efficient state $\mu^{* *}=\left(m_{1} \delta_{\alpha_{1}^{* *}}, \cdots, m_{n} \delta_{\alpha_{n}^{* *}}\right)$, where $\left(\alpha_{1}^{* *}, \cdots, \alpha_{n}^{* *}\right) \in$ $\prod_{p=1}^{n}[\underline{x}, \bar{x}]$ is the unique maximizer of $\bar{G}$ defined in (10).

Proposition 3.5, therefore, provides a convenient way to characterize the efficient state of the aggregative game (7). It shows that the efficient state of the aggregative game is in monomorphic population states. Every agent in population $p$ plays $\alpha_{p}^{* *}$, where $\left(\alpha_{1}^{* *}, \cdots, \alpha_{n}^{* *}\right)$ is the unique maximizer of $\bar{G}$. The fact that we can use $\bar{G}$ to characterize the efficient state is a feature of aggregative games. In general, maximizing $\bar{F}$ is difficult due to its measure theoretic character. But due to the monomorphic nature of the efficient state, and because $F$ is an aggregative game, we can capture the effect of a population state $\mu_{p}$ through the scalar $m_{p} \alpha_{p}$. This is the population level aggregate strategy when all agents in the population are playing $\alpha_{p}$. Thus, the aggregative nature of $F$ provides an easily implementable way to compute the efficient state, which is one of the reasons why we have focused on such games. ${ }^{10}$

As an illustration, we apply this result to the public goods model in Example 3.2. Since $\beta_{p}(x, \alpha)=v_{p}(\alpha)-c_{p}(x)$ in this example, the $\bar{G}$ function takes the specific form

$$
\bar{G}\left(\alpha_{1}, \cdots, \alpha_{n}\right)=\sum_{p \in \mathcal{P}} m_{p} v_{p}\left(\sum_{q \in \mathcal{P}} m_{q} \alpha_{q}\right)-\sum_{p \in \mathcal{P}} m_{p} c_{p}\left(\alpha_{p}\right) .
$$

Lahkar and Mukherjee [16] provide an analysis of this function and characterize the efficient state of this game. Here, our purpose is to derive those results as a consequence of the more general Proposition 3.5. We need to check that Assumptions 3.1 and 3.3 are satisfied by this game. Assumption 3.1 follows readily due to the strict convexity of $c_{p}$, for every $p$, in Example 3.2. The proof of Lemma 3.3 in Lahkar and Mukherjee [16] shows that Assumption 3.3 is also satisfied. Hence, Proposition 3.5 holds for the public goods model.

\section{Evolutionary Implementation}

We now introduce a planner who, given any type distribution $m=\left(m_{1}, m_{2}, \cdots, m_{n}\right)$, wishes to implement the efficient state $\mu^{* *}$ corresponding to that type distribution as characterized in Proposition 3.5. In the conventional terminology of mechanism design theory, the mapping $m \mapsto \mu^{* *}$ is the planner's social choice function in the aggregative game $F$ defined by (7). We assume

\footnotetext{
${ }^{10}$ We could have avoided the measure theoretic complications by considering a finite approximation of the strategy set $\mathcal{S}$. In that case, however, it would be difficult to precisely characterize the efficient state of $F$. Maximizing $\bar{G}$ would provide only an approximation as the maximizer $\alpha_{p}^{*}$ may not belong to the finite approximation of $\mathcal{S}$ for some population $p$. The elegance imparted by this exact characterization is the major reason why we opt for a continuous strategy approach. Further details are in Appendix A.2.1 of Lahkar and Mukherjee [16].
} 
that the planner does not know the type of individual agents. This makes our implementation problem a non-trivial one because the payoffs in $F$ are type or population specific. ${ }^{11}$ Our approach to implementation is, however, not the classical one of a direct mechanism through which the planner induces truthful revelation of types and then assigns optimal strategies and transfers to agents. Instead, we adopt the methodology of evolutionary implementation wherein strategy choices continue to be made by agents but is guided towards the efficient state dynamically by the planner through appropriate transfer pricing. At the end of this section, we provide some comments about the informational requirements for this approach.

Before proceeding further, we provide a more careful comparison of payoffs in our model with those in Sandholm [33, 34], who refer to type specific payoffs as idiosyncratic payoffs. Payoffs in those papers are separable into a common part which is the same for all agents in the society and an idiosyncratic part that is type specific. Thus, if we had applied that model of payoffs into our context, then that would have implied that all agents have the same base payoff $\beta(x, A(\mu))$ instead of diverse payoffs $\beta_{p}(x, A(\mu))$ as is the case in (7). We would then have incorporated type specific heterogeneity by introducing a parameter $\theta_{x, p}$ which would have been the idiosyncratic payoff of a type $-p$ agent playing strategy $x$. Thus, total payoff of such an agent would have been $F_{x, p}(\mu)=\beta(x, A(\mu))+\theta_{x, p} \cdot{ }^{12}$ Our entire analysis of evolutionary implementation would have go through with this interpretation. However, we believe that in economic applications like the public goods/bads model or the tragedy of the commons, it is more meaningful to introduce heterogeneity directly into the base payoff instead of through a separate parameter $\theta_{x, p}$. Such heterogeneity can then be given a more substantive interpretation as arising from differences in valuation or cost functions. This is why we have chosen to make our model of payoffs different from Sandholm $[33,34]$ and make the basic payoff function $\beta_{p}(x, A(\mu))$ type specific or idiosyncratic. Of course, that is no way detracts from the fact that the fundamental notion of evolutionary implementation underlying this paper originates in Sandholm's papers.

The aggregative game (7) has certain convenient properties which make it amenable for evolutionary implementation. First, it has a unique efficient state. Second, the aggregate payoff function (8) is concave. These features will allow the planner to globally implement the efficient state in this model. To implement the efficient state, we allow the planner to create a variable externality pricing scheme which is a transfer scheme that equals the total externality imposed an agent. To construct such a transfer scheme, we apply (3) to first calculate externalities in the aggregative game (7). The following proposition presents the result. The proof is in Appendix A.3.

Proposition 4.1 Consider the aggregative game $F$ as defined in (7). The total externality imposed

\footnotetext{
${ }^{11} \mathrm{Had}$ the planner known the type of individual agents, then he could have simply assigned the efficient strategy $\alpha_{p}^{* *}$ characterized in Proposition 3.5 to type $-p$ agents.

${ }^{12}$ Thus, under this interpretation, the payoff in the public goods game would have been $F_{x, p}(\mu)=v(A(\mu))-c(x)+$ $\theta_{x, p}$.
} 
by agents in population $p$ who play $x \in \mathcal{S}$ in this game is

$$
e_{x, p}(\mu)=x \sum_{q \in \mathcal{P}} \int_{\mathcal{S}} \beta_{q, 2}(y, A(\mu)) \mu_{q}(d y)
$$

where $\beta_{q, 2}(y, \alpha)=\frac{\partial \beta_{q}(y, \alpha)}{\partial \alpha}$.

Thus in Example 3.2, $\beta_{q, 2}(y, A(\mu))=v_{q}^{\prime}(A(\mu))$. Using (12), we can then calculate externalities in the public goods game as $e_{x, p}(\mu)=x \sum_{q \in \mathcal{P}} \int_{\mathcal{S}} v_{q}^{\prime}(A(\mu)) \mu_{q}(d y)=x \sum_{q \in \mathcal{P}} m_{q} v_{q}^{\prime}(A(\mu))$. This is as calculated in Proposition 3.5 of Lahkar and Mukherjee [16]. Here, it follows as a consequence of the more general Proposition 4.1.

Using $t_{x, p}(\mu)$ to denote the transfer to such agents at $\mu$, we define the variable externality pricing scheme (Sandholm [32]) in the aggregative game $F$ as

$$
t_{x, p}(\mu)=e_{x, p}(\mu)=x \sum_{q \in \mathcal{P}} \int_{\mathcal{S}} \beta_{q, 2}(y, A(\mu)) \mu_{q}(d y),
$$

where the second equality comes from (12). Thus, the transfer is equal to the externality generated by agents. By imposing this transfer on agents in the aggregative game $F$, the planner creates a new game $\hat{F}$ in which payoffs take the form

$$
\begin{aligned}
\hat{F}_{x, p}(\mu) & =\beta_{p}(x, A(\mu))+t_{x, p}(\mu) \\
& =\beta_{p}(x, A(\mu))+x \sum_{q \in \mathcal{P}} \int_{\mathcal{S}} \beta_{q, 2}(y, A(\mu)) \mu_{q}(d y) .
\end{aligned}
$$

By the definition in (13), $t_{x, p}(\mu)=e_{x, p}(\mu)$. Therefore, formally, (14) is equivalent to the externality adjusted game (4) with $F$ being the aggregative game (7). Hence, we refer to $\hat{F}$ defined by (14) as the externality adjusted aggregative game. Intuitively, the imposition of this transfer means that in the new game $\hat{F}$, agents internalize the externality they create in the original aggregative game $F$. We now obtain the following result characterizing the unique Nash equilibrium of $\hat{F}$.

Theorem 4.2 The externality adjusted aggregative game $\hat{F}$ defined by (14) is a potential game with potential function $\bar{F}$ defined by (8). Further suppose that the aggregative game $F$ satisfies Assumptions 3.1 and 3.3. Then, $\hat{F}$ has a unique Nash equilibrium $\mu^{* *}$, which is the unique efficient state of the aggregative game $F$ as characterized in Proposition 3.5.

Proof. The conclusion that $\hat{F}$ is a potential game with potential function $\bar{F}$ arises from Proposition 2.4 and from the fact that $t_{x, p}(\mu)=e_{x, p}(\mu)$ as defined in (13). Under Assumptions 3.1 and 3.3, Lemma 3.4 shows that $\bar{F}$ is concave with a unique maximizer. If the potential function is concave, then Nash equilibria of the underlying potential game coincide with the maximizers of the potential function (Sandholm [31], Cheung and Lahkar [5]). By Proposition 3.5, the unique maximizer of $\bar{F}$ is $\mu^{* *}$. Hence, it is the unique Nash equilibrium of $\hat{F}$. But by definition, the maximizer of $\bar{F}$ is the efficient state of $F$. 
For the public goods game in Example 3.2, we calculated $e_{x, p}(\mu)=x \sum_{q \in \mathcal{P}} m_{q} v_{q}^{\prime}(A(\mu))$. Therefore, with $t_{x, p}(\mu)=e_{x, p}(\mu)$, the externality adjusted game (14) for this model takes the form

$$
\hat{F}_{x, p}(\mu)=v_{p}(A(\mu))+x \sum_{q \in \mathcal{P}} m_{q} v_{q}^{\prime}(A(\mu))-c_{p}(x)
$$

Lahkar and Mukherjee [16] provide a detailed analysis of this externality adjusted game. Here, we have already verified that this game satisfies Assumptions 3.1 and 3.3. Therefore, it follows as a consequence of Theorem 4.2 that this game has a unique Nash equilibrium which is the efficient state of the original public goods game.

Theorem 4.2 shows that the variable externality price scheme $t_{x, p}(\mu)$ implements efficiency in equilibrium in aggregative games of the form (7). Evolutionary implementation, however, seeks not just to show implementation in equilibrium but also convergence to the efficient state from arbitrary initial states. Lahkar and Mukherjee [16] analyze evolutionary implementation in the continuous-strategy public goods game. Here, we seek to extend that analysis to all aggregative games of the form (7). For this purpose, we consider five canonical dynamics from evolutionary game theory. These are the replicator dynamic (Oechssler and Riedel [23, 24], Cheung [4]), the the Brown-von Neumann-Nash (BNN) dynamic (Hofbauer et al. [12]), the pairwise comparison dynamic (Cheung [3]), the logit dynamic (Perkins and Leslie [26], Lahkar and Riedel [14]) and the best response dynamic (Lahkar and Mukherjee [16]). ${ }^{13}$

Apart from the best response dynamic, the other four evolutionary dynamics have been extended to general continuous-strategy games. To define these dynamics for the externality adjusted aggregative game (14), we introduce the average payoff in population $p$ at social state $\mu$, which is $\overline{\hat{F}}_{p}(\mu)=\frac{1}{m_{p}} \int_{\mathcal{S}} \hat{F}_{x, p}(\mu) \mu_{p}(d x)$. This leads to the notion of the excess payoff of a strategy $x$, $\hat{F}_{x, p}(\mu)-\overline{\hat{F}}_{p}(\mu)$, which will be required in the definitions of the replicator dynamic and the BNN dynamic. In addition, for the logit dynamic, we also require the probability measure $L_{\eta, p}(\mu)$ on $\mathcal{S}$, which we define as $L_{\eta, p}(\mu)(T)=\int_{T} \frac{\exp \left(\eta^{-1} \hat{F}_{x, p}(\mu)\right)}{\int_{\mathcal{S}} \exp \left(\eta^{-1} \hat{F}_{y, p}(\mu)\right) d y} d x, T \subseteq \mathcal{S}, \eta>0$. In the logit dynamic, we interpret the parameter $\eta$ as a perturbation parameter. We explain its relevance further below. This probability measure $L_{\eta, p}(\mu)$ is called the logit choice measure for population $p$ given $\eta$ and the current social state $\mu$. Intuitively, $L_{\eta, p}(\mu)$ is an approximation of the best response in the sense that for $\eta$ small, it puts most of the probability mass on the set of best responses to $\mu$. It is generated when agents best respond to a perturbed version of payoffs, where the perturbation depends upon $\eta$ (Lahkar and Riedel [14]).

We now define the replicator dynamic, the BNN dynamic, the pairwise comparison dynamic and the logit dynamic respectively in $\hat{F}$ defined by (14) as follows.

$$
\dot{\mu}_{p}(T)=\int_{T}\left(\hat{F}_{x, p}(\mu)-\overline{\hat{F}}_{p}(\mu)\right) \mu_{p}(d x)
$$

\footnotetext{
${ }^{13}$ The papers cited in the context of these dynamics here analyze the continuous-strategy versions of these dynamics. These are the versions that are relevant for us. All these dynamics were originally developed in the context of finite strategy population games. Sandholm [35] provides a detailed textbook analysis of the finite-strategy version of these dynamics.
} 


$$
\begin{aligned}
& \dot{\mu}_{p}(T)=m_{p} \int_{T}\left[\hat{F}_{x, p}(\mu)-\overline{\hat{F}}_{p}(\mu)\right]_{+} d x-\mu_{p}(T) \int_{\mathcal{S}}\left[\hat{F}_{y, p}(\mu)-\overline{\hat{F}}_{p}(\mu)\right]_{+} d y, \\
& \dot{\mu}_{p}(T)=\int_{\mathcal{S}} \int_{T}\left[\hat{F}_{x, p}(\mu)-\hat{F}_{y, p}(\mu)\right]_{+} d x \mu_{p}(d y)-\int_{\mathcal{S}} \int_{T}\left[\hat{F}_{y, p}(\mu)-\hat{F}_{x, p}(\mu)\right]_{+} \mu_{p}(d x) d y, \\
& \dot{\mu}_{p}(T)=m_{p} L_{\eta, p}(\mu)(T)-\mu_{p}(T), \text { where } \eta>0 .
\end{aligned}
$$

In each of these cases, $\dot{\mu}_{p}(T)$ represents the direction and magnitude of change in the mass of agents in population $p$ who are playing strategies in $T \subseteq \mathcal{S}$. Under the replicator dynamic (16), the mass of agents playing strategies in $T$ increases if the aggregate excess payoff of such strategies is positive. The BNN dynamic (17) involves agents adopting strategy $x$ with probability proportional to the positive part of the excess payoff $F_{x, p}(\mu)-\overline{\hat{F}}_{p}(\mu)$ of that strategy (note that $\left.[a-b]_{+}=\max (a-b, 0)\right)$. Under the pairwise comparison dynamic, agents abandon strategy $y$ and adopt strategy $x$ with probability proportional to $\left[\hat{F}_{x, p}(\mu)-\hat{F}_{y, p}(\mu)\right]_{+}$. In the logit dynamic, the social state $\mu$ moves towards the logit choice measure $L_{\eta, p}(\mu)$.

Unlike the four dynamics defined in (16)-(19), the best response (BR) dynamic cannot in general be defined for all continuous-strategy games. The best response may not even exist in such games at certain social states. Even if it does, the best response may be multi-valued making the technical analysis of the resulting dynamic difficult. Lahkar and Mukherjee [16], however, show that these problems do not occur in aggregative games. In such games, the best response always exists and is, in fact, uniquely defined at all social states. This makes it feasible to define the BR dynamic for such games and analyze it using standard techniques of evolutionary dynamics on games with continuous strategy sets. To define this dynamic for the externality adjusted aggregative game (14), we make the following assumption.

Assumption 4.3 The externality adjusted aggregative game $\hat{F}$ defined by (14) is also an aggregative game.

Assumption 4.3, therefore, requires that the payoffs in $\hat{F}$ depend only upon the individual strategy and the aggregate strategy level $A(\mu)$. Given that the original game (7) is already aggregative, the satisfaction of this assumption requires that $\sum_{q} \int_{\mathcal{S}} \beta_{q, 2}(y, A(\mu)) \mu_{q}(d y)$ in (14) is a function of $A(\mu)$. Thus, in the public goods game (Example 3.2), we calculated $\sum_{p} \int_{\mathcal{S}} \beta_{q, 2}(y, A(\mu)) \mu_{q}(d y)=$ $\sum_{p} m_{p} v_{p}^{\prime}(A(\mu))$. Hence, its externality adjusted extension (15) is also an aggregative game.

In addition, the strict concavity of $\beta_{p}(x, A(\mu))$ with respect to $x$ (Assumption 3.1 ) implies that $(14)$ is also strictly concave in $x$. Hence, given $A(\mu)$, there exists a unique maximizer to (14) in $\mathcal{S}$. This is the unique best response of an agent in population $p$ to social state $\mu$ in $\hat{F}$. ${ }^{14}$ Since $\hat{F}$ is an aggregative game by Assumption 4.3, social states which generate the same aggregate strategy level will lead to the same best response. Therefore, if $A(\mu)=\alpha$, we denote the best response of

\footnotetext{
${ }^{14}$ The existence of such a unique best response applies not just to $\hat{F}$ but to any aggregative game where payoffs are strictly concave with respect to individual strategy $x$.
} 
an agent in population $p$ at the state $\mu$ in $\hat{F}$ as $b_{p}(\alpha)$ and define it formally as

$$
b_{p}(\alpha)=\arg \max _{x \in \mathcal{S}} \hat{F}_{x, p}(\mu) \text { for all } \mu \in \Delta \text { such that } A(\mu)=\alpha \in[\underline{x}, \bar{x}]
$$

The BR dynamic for an aggregative game like $\hat{F}$ in which every social state generates a unique best response then takes the form

$$
\dot{\mu}_{p}=m_{p} \delta_{b_{p}(\alpha)}-\mu_{p}
$$

where $b_{p}(\alpha)$ is the unique best response of an agent in population $p$ to $\mu$ as defined in (20). Thus, under this dynamic, every population state moves towards the monomorphic state where every agent is playing the best response to the current state.

Before stating our key result on evolutionary implementation, we note that in the BNN dynamic (17), the pairwise comparison dynamic (18) and the BR dynamic (21), the set of rest points coincide with the set of Nash equilibria of the underlying population game. Hence, in $\hat{F}$, these dynamics would have a unique rest point, which is the Nash equilibrium $\hat{\mu}^{* *}$. In the replicator dynamic (16), the set of Nash equilibria form a subset of the set of rest points. ${ }^{15}$ The set of rest points in the logit dynamic (19) is the set of logit equilibria, which, for $\eta$ small, are approximations of Nash equilibria (Lahkar and Riedel [14]).

We now state the following proposition on evolutionary implementation. The key to this result is that the externality adjusted aggregative game is a potential game. This implies convergence of evolutionary dynamics to its Nash equilibrium, which is also the efficient state of the original aggregative game (Theorem 4.2). The result, therefore implies that once the planner has made agents internalize the externality they create through the variable externality pricing scheme (13), society converges to the unique efficient state of $F$ under a wide variety of evolutionary dynamics.

Theorem 4.4 Consider the externality adjusted aggregative game $\hat{F}$ as defined in (14) and let Assumptions 3.1 and 3.3 be satisfied. Hence, $\hat{F}$ has a unique Nash equilibrium $\mu^{* *}$ as characterized in Theorem 4.2, which is also the unique efficient state of the aggregative game F as defined in (7). Then,

1. From every initial state $\mu(0)$ in the interior of $\Delta$, the replicator dynamic (16) converges to $\mu^{* *}$.

2. From every initial state $\mu(0)$ in $\Delta$, the BNN dynamic (17) and the pairwise comparison dynamic (18) converges to $\mu^{* *}$.

3. Let $\Delta_{D} \subset \Delta$ to be a set of social states $\mu=\left(\mu_{1}, \cdots, \mu_{n}\right)$ such that for every $p$, $\mu_{p}$ has a bounded density function and $\left(m_{1} L_{\eta, 1}(\mu), \cdots, m_{n} L_{\eta, n}(\mu)\right) \in \Delta_{D}$ for every $\mu \in \Delta$. Then, from every initial state $\mu(0) \in \Delta_{D}$, the logit dynamic (19) converges to a logit equilibrium of $\hat{F}$. For $\eta$ small, any such logit equilibrium approximates (in distribution) the unique Nash equilibrium $\mu^{* *}$ of $\hat{F}$.

\footnotetext{
${ }^{15}$ It is well known that non-Nash social states in monomorphic population states are also rest points of the replicator dynamic.
} 
4. In addition to Assumptions 3.1 and 3.3, let Assumption 4.3 be also satisfied. Then, from every initial state $\mu(0)$ in $\Delta$, the BR dynamic (21) converges to $\mu^{* *}$.

In each case, convergence means that the limit point of the trajectory (in distribution) is $\mu^{* *}$ or, in the case of the logit dynamic, a logit equilibrium.

Proof. Recall from Theorem 4.2 that $\hat{F}$ is a potential game with a unique Nash equilibrium $\mu^{* *}$. Hence, in each case, the conclusion follows from relevant results on convergence in potential games. Thus, convergence under (1) the replicator dynamic follows from Oechssler and Riedel $[23,24]$ and Cheung [4], (2) the BNN dynamic and the Smith dynamic follow from Hofbauer et al. [12] and Cheung [3] respectively, (3) the logit dynamic from Lahkar and Riedel [14] and (4) the BR dynamic from Lahkar and Mukherjee [16]. The BR dynamic also requires the fulfilment of Assumption 4.3 to ensure that this dynamic is well-defined on $\hat{F}$.

Theorem 4.4 is our main result and is a generalization of Theorem 5.1 and Proposition 5.2 in Lahkar and Mukherjee [16] on the public goods game to all aggregative games that satisfy Assumptions 3.1 and 3.3. The key to this result is that the externality adjusted aggregative game $\bar{F}$ is a potential game. Hence, existing results on convergence in potential games imply each of the statements in the proposition. For the BNN dynamic, the Smith dynamic and the BR dynamic, convergence to $\mu^{* *}$ is global as their unique rest point is $\mu^{* *}$. For the replicator dynamic, we need to restrict initial states to the interior of $\Delta$ as non-Nash social states in monomorphic population states are also rest points of this dynamic. For these dynamics, convergence happens because the potential function $\bar{F}$ (see Theorem 4.2) itself acts as the Lyapunov function along which solution trajectories ascend as they converge to $\mu^{* *}$. For the logit dynamic, certain technical reasons related to the existence of directional derivatives require that we only allow initial states with bounded density functions. The set $\Delta_{D}$ described in part 3 of this proposition is such a set. The existence of such a set has been established by Perkins and Leslie [26]. We also need to modify the potential function into an "entropy-adjusted potential function" to establish convergence (Lahkar and Riedel [14]). Furthermore, convergence under the logit dynamic is to a logit equilibrium. For $\eta$ small, any such logit equilibrium must approximate a Nash equilibrium of the underlying game which, in the case of $\hat{F}$, is $\mu^{* *}$ (Lahkar and Riedel [14]). We also note that convergence under the replicator, $\mathrm{BNN}$, pairwise comparison and logit dynamics is independent of Assumption 4.3. All we require for global evolutionary implementation in our aggregative game model under these dynamics is that $\hat{F}$ has a unique Nash equilibrium, which follows from Assumptions 3.1 and 3.3 (see Proposition 3.5 and Theorem 4.2). Assumption 4.3 is only required for the BR dynamic to ensure that $\hat{F}$ has a unique Nash equilibrium at every social state.

We now obtain the main conclusion of Lahkar and Mukherjee [16] as an application of Theorem 4.4. That paper establishes the evolutionary implementation of the efficient state of the public goods model we have described in Example 3.2 (see Theorem 5.1 and Proposition 5.2 in that paper). As part of our general analysis, we have already verified that example satisfies Assumptions 3.1, 3.3 and 4.3. Hence, Theorem 4.4 applies to that model. In the next section, we consider two additional 
applications of this result. These are to a model of public bads and to a model of the tragedy of the commons.

We conclude this section with a discussion of what the planner needs to know to evolutionarily implement $\mu^{* *}$. Clearly, this requires the planner to calculate the total externality (12). Notice that externality is common for all strategy $x$ users so that the planner does not need to know the type of individual agents in calculating it, which is what we have assumed. But there may be other informational requirements depending upon the structure of the payoff function (7). It is possible that the total externality does not depend upon type-specific components of the payoffs. For example, in the public goods game from Example 3.2, suppose only the cost function differs across types while the benefit function is common to all types and equals $v(A(\mu))$. In that case, it is clear from (15) that the total externality would equal $x \sum_{p} v^{\prime}(A(\mu))$. The planner, in that case, would only need to observe the individual strategy $x$ and the aggregate strategy level $A(\mu)$ and know the common benefit function $v$. In particular, there would be no need to know the type distribution $m .{ }^{16}$ This is similar to the requirements in classical mechanisms like the VCG mechanism that leads to dominant strategy implementation. This is also the case, for example, in Sandholm [33, 34] where payoffs have a common component and an idiosyncratic or type-specific component (see footnote 11) and in which, externalities arise from the common component.

If, however, the externality does depend upon the type-specific component of payoffs, then the informational requirements for computing externalities using the parameters of the model are more onerous. In this general case, the planner would also need to know the type distribution $m$ along with the type-specific payoff components. This is most clearly seen in the externality calculated in (15) for the public goods game. Of course, allowing the planner to have knowledge such extensive knowledge of different payoff functions and the type distribution is definitely a stronger assumption than in Sandholm $[33,34]$. But in certain parts of the mechanism design literature, such assumptions are routine. ${ }^{17}$

But even if the planner does not have knowledge of payoff functions and the type distribution, it may still be possible to apply evolutionary implementation. After all, evolutionary implementation depends upon the current externality level, which can be empirically estimated. As we also discuss in Lahkar and Mukherjee [16], there are well-established empirical techniques to calculate externalities (see, for example, Lin [20]). Such empirical techniques include the instrumental variable method (Ayres and Levitt [1]), hedonic pricing method (Li and Brown [19], Chay and Greenstone [2], Le Goffe [18]) and contingent valuation method (Hanemann [11]). The planner should, therefore, be able to estimate externalities empirically in our model and apply evolutionary implementation even if knowledge of different payoff functions and their distribution is not available.

\footnotetext{
${ }^{16} \mathrm{In}$ fact, this is the case in Sandholm [33, 34]. The aggregate social state appears only in the common part of payoffs so that externalities arise entirely from that part. The idiosyncratic part depends entirely upon individual strategy and, hence, plays no role in determining externalities. Therefore, the planner has no need to know the type distribution to know externalities. See also Footnote 11.

${ }^{17}$ See, for example, the literature on Bayesian Nash implementation (Maskin and Sjöström [21]).
} 


\section{$5 \quad$ Further Applications}

\subsection{A Model of Public Bads}

We define the aggregative game $F$ in which payoffs take the form

$$
F_{x, p}(\mu)=v_{p}(x)-c_{p}(A(\mu)) .
$$

We interpret $v_{p}: \mathcal{S} \rightarrow \mathbf{R}$ as the benefit function for an agent in population $p$ and $c_{p}:[\underline{x}, \bar{x}] \rightarrow \mathbf{R}$ as the cost function for that agent. Thus, the benefit for an agent depends upon the agent's own strategy while the cost depends upon the aggregate strategy level. This model is, therefore, the reverse of the public goods game considered in Lahkar and Mukherjee [16] (Example 3.2). Here, the benefit is private and the cost is social. We, therefore, refer to (22) as a model of public bads. One situation in which this model might be relevant is when $x$ represents the level of pollution that an agent may generate.

The public bads game is clearly an aggregative game. In terms of the general definition of an aggregative game (7), this game is characterized by $\beta_{p}(x, \alpha)=v_{p}(x)-c_{p}(\alpha)$ for $x, \alpha \in[\underline{x}, \bar{x}]$. We assume that both $v_{p}$ and $c_{p}$ are strictly increasing. Further, in keeping with Assumption 3.1, we assume that $v_{p}$ is strictly concave. We also assume that $c_{p}$ is strictly convex. This will ensure the satisfaction of Assumption 3.3.

Calculating the Nash equilibrium of the public bads game is easy. Note from (22) that the individual strategy $x$ of an agent only affects the benefit $v_{p}(x)$, which is strictly increasing in $x$. Therefore, for every $\mu \in \Delta, \arg \max _{x \in \mathcal{S}} F_{x, p}(\mu)=\bar{x}$. Hence, $\bar{x}$ is the dominant strategy for every agent in every population in this game. The Nash equilibrium and, in fact, the dominant strategy equilibrium, of this model is, therefore, $\mu^{*}=\left(m_{1} \delta_{\bar{x}}, \cdots, m_{n} \delta_{\bar{x}}\right)$. Every agent at that Nash equilibrium plays $\bar{x}$, the highest possible strategy.

To characterize the efficient state in this game, we compute its aggregate payoff to be

$$
\begin{aligned}
\bar{F}(\mu) & =\sum_{p \in \mathcal{P}} \int_{\mathcal{S}} F_{x, p}(\mu) \mu_{p}(d x) \\
& =\sum_{p \in \mathcal{P}} \int_{\mathcal{S}}\left(v_{p}(x)-c_{p}(A(\mu))\right) \mu_{p}(d x) \\
& =\sum_{p \in \mathcal{P}} \int_{\mathcal{S}} v_{p}(x) \mu_{p}(d x)-\sum_{p \in \mathcal{P}} m_{p} c_{p}(A(\mu)) .
\end{aligned}
$$

Thus, an efficient state in this game maximizes (23). To characterize such an efficient state, we define the corresponding $\bar{G}$ function as

$$
\bar{G}\left(\alpha_{1}, \cdots, \alpha_{n}\right)=\sum_{p \in \mathcal{P}} m_{p} v_{p}\left(\alpha_{p}\right)-\sum_{p \in \mathcal{P}} m_{p} c_{p}\left(\sum_{q \in \mathcal{P}} m_{q} \alpha_{q}\right) .
$$


The following result characterizes the efficient state of this game. Details of the proof, which follows from Proposition 3.5, is in Appendix A.4.

Proposition 5.1 Consider the public bads game $F$ defined by (22). This game has a unique efficient state $\mu^{* *}=\left(m_{1} \delta_{\alpha_{1}^{* *}}, \cdots, m_{n} \delta_{\alpha_{n}^{* *}}\right)$, where $\left(\alpha_{1}^{* *}, \cdots, \alpha_{n}^{* *}\right) \in \prod_{p=1}^{n}[\underline{x}, \bar{x}]$ is the unique maximizer of $\bar{G}$ defined in (24).

In order to discuss implementation of this efficient state, we need to calculate externalities in the public bads game. Recall that $\beta_{p}(x, \alpha)=v_{p}(x)-c_{p}(\alpha)$ in this model. Hence, applying Proposition 4.1 , we arrive at the following result.

Corollary 5.2 Consider the public bads game $F$ defined by (22). The total externality imposed by an agent in population $p$ who plays $x \in \mathcal{S}$ in $F$ at $\mu \in \Delta$ is

$$
e_{x, p}(\mu)=-x \sum_{q \in \mathcal{P}} m_{q} c_{q}^{\prime}(A(\mu))
$$

Moreover, $e_{x, p}(\mu)<0$. Therefore, externalities are negative in the public bads model.

Proof. Since $\beta_{q}(x, \alpha)=v_{q}(x)-c_{q}(\alpha)$ in $F, \beta_{q, 2}(y, A(\mu))=-c_{q}^{\prime}(A(\mu))$. Hence, (12) follows from Proposition 4.1. Negative externalities follow from the strict convexity of $c_{p}$.

We now allow the planner to introduce the variable externality pricing scheme $t_{x, p}(\mu)=e_{x, p}(\mu)$ as defined in (13). Since, by Corollary 5.2, externalities are negative, the transfer under this scheme, is a tax on agents equal to the total negative externality they impose on society. Following (14), (22) and (25), payoffs in the externality adjusted public bads game take the form

$$
\begin{aligned}
\hat{F}_{x, p}(\mu) & =F_{x, p}(\mu)+t_{x, p}(\mu) \\
& =v_{p}(x)-c_{p}(A(\mu))-x \sum_{q \in \mathcal{P}} m_{q} c_{q}^{\prime}(A(\mu)) .
\end{aligned}
$$

Clearly, $\hat{F}$ is also an aggregative game. Hence, this model also satisfies Assumption 4.3. ${ }^{18}$ We, therefore, obtain our main result for the public bads application.

Proposition 5.3 Consider the externality adjusted public bads game $\hat{F}$ defined in (26) obtained through the variable externality pricing scheme $t_{x, p}(\mu)=e_{x, p}(\mu)$, where $e_{x, p}(\mu)$ is as characterized in (25). This game has a unique Nash equilibrium $\mu^{* *}$, which is the unique efficient state of the public bads game $F$ as characterized in Proposition 5.1. Further, the replicator dynamic (16), the $B N N$ dynamic (17), the pairwise comparison dynamic (18) and the BR dynamic (21) evolutionarily implement $\mu^{* *}$ as per Theorem 4.4.

\footnotetext{
${ }^{18}$ Thus, $\sum_{p} \int_{\mathcal{S}} \beta_{q}(y, A(\mu)) \mu_{q}(d y)=-\sum_{p} m_{p} c_{p}^{\prime}(A(\mu))$ in the public bads model.
} 
Proof. We have verified in the proof of Proposition 5.1 that the public goods game $F$ satisfies Assumptions 3.1 and 3.3. It is also evident from (26) that this game satisfies Assumption 4.3. Hence, the characterization of $\mu^{* *}$. The conclusions in the proposition, therefore, follow from Theorems 4.2 and 4.4 .

\subsection{Tragedy of the Commons}

The second application we consider is the tragedy of the commons. We interpret $x \in \mathcal{S}$ as be a specific level of input that an agent uses in a common pool resource, where we assume that $\underline{x}>0 .{ }^{19}$ The aggregate strategy level (6) then becomes the aggregate input level which generates total output $\pi(A(\mu))$, where $\pi: \mathbf{R}_{+} \rightarrow \mathbf{R}_{+}$is a strictly increasing production function. We assume that $\pi$ is strictly concave and that the average product of $\pi, A P(A(\mu))=\frac{\pi(A(\mu))}{A(\mu)}$, is strictly declining for all $A(\mu) \in[\underline{x}, \bar{x}]$. Since the resource is a common pool resource, we define the payoff of an agent in population $p$ playing $x \in \mathcal{S}$ to be

$$
\begin{aligned}
F_{x, p}(\mu) & =x \frac{\pi(A(\mu))}{A(\mu)}-c_{p}(x) \\
& =x A P(A(\mu))-c_{p}(x)
\end{aligned}
$$

where $c_{p}(x)$ is the cost of using input level $x$ for an agent of type $p$.

We refer to the game $F$ defined by (27) as the large population tragedy of the commons model with multiple types. It is an extension of the single population tragedy of the commons model analyzed in Cheung and Lahkar [5], although not from the point of view of evolutionary implementation. This model clearly constitutes an aggregative game of the form (7) with $\beta_{p}(x, A(\mu))=x A P(A(\mu))-c_{p}(x)$. In order to satisfy Assumption 3.1, we assume $c_{p}$ to be strictly convex on $[\underline{x}, \bar{x}]$. The strict concavity of the production function will ensure satisfaction of Assumption 3.3 .

We first seek to characterize the Nash equilibrium of this model. In the public bads model, this was easy due to the presence of a dominant strategy. In the present case, however, there is no such dominant strategy. Instead, we apply the general method developed in Lahkar and Sultana [17] to characterize Nash equilirbia of such games. We present further details of that approach in Appendix A.4.1. The first step in that approach is to show that the tragedy of the commons itself constitutes a potential game. The proof is in Appendix A.4.1.

Proposition 5.4 The tragedy of the commons F defined by (27) is a potential game with potential function $f: \mathcal{M} \rightarrow \mathbf{R}$ defined by

$$
f(\mu)=\int_{\underline{x}}^{A(\mu)} A P(z) d z-\sum_{p \in \mathcal{P}} c_{p}(x) \mu_{p}(d x) .
$$

\footnotetext{
${ }^{19}$ We require $\underline{x}>0$ to ensure that the average product in $(27)$ is bounded on $[\underline{x}, \bar{x}]$. If $\underline{x}=0$, then $A P(z)$ would no longer be bounded. The assumption $\underline{x}>0$ is without too much of a loss of generality as it can be arbitrarily close to zero.
} 
Further, the potential function is concave but not strictly concave on $\Delta$. Hence, the set of Nash equilibria of $F$ coincide with the set of maximizers of $f$, which is a convex set.

The concavity of the potential function (28) means that we can characterize any Nash equilibrium by maximizing this function. As usual, maximizing $f$ directly is difficult due to its measure theoretic context. Instead we construct its quasi-potential function (Lahkar [15], Cheung and Lahkar [5]). ${ }^{20}$ This leads to the following result. The proof is in Appendix A.4.1.

Proposition 5.5 The tragedy of the commons $F$ defined by (27) has a unique Nash equilibrium $\mu^{*}=\left(m_{1} \delta_{\alpha_{1}^{*}}, m_{2} \delta_{\alpha_{2}^{*}}, \cdots, m_{n} \delta_{\alpha_{n}^{*}}\right)$, where $\left(\alpha_{1}^{*}, \cdots, \alpha_{n}^{*}\right) \in \prod_{p \in \mathcal{P}}[\underline{x}, \bar{x}]$ is the unique maximizer of the quasi-potential function $g: \prod_{p=1}^{n}[\underline{x}, \bar{x}] \rightarrow \mathbf{R}$ defined as

$$
g\left(\alpha_{1}, \cdots, \alpha_{n}\right)=\int_{\underline{x}}^{\sum_{p \in \mathcal{P}} m_{p} \alpha_{p}} A P(z) d z-\sum_{p \in \mathcal{P}} m_{p} c_{p}\left(\alpha_{p}\right) .
$$

Intuitively, (5.5) implies that $\mu^{*}$ is characterized by $A P\left(A\left(\mu^{*}\right)\right)=c_{p}^{\prime}\left(\alpha_{p}^{*}\right)$, where $A\left(\mu^{*}\right)=$ $\sum_{p} m_{p} \alpha_{p}^{*}{ }^{21}$ Therefore, at the unique Nash equilibrium of the tragedy of the commons, every agent equates the average product of the aggregate input level to their individual marginal cost. This suggests the presence of inefficiency in equilibrium as efficiency would require equating marginal product to marginal cost. We verify this argument formally by defining the aggregate payoff in this model. The aggregate payoff in the tragedy of the commons (27) at a state $\mu$ is

$$
\begin{aligned}
\bar{F}(\mu) & =\sum_{p \in \mathcal{P}} \int_{\mathcal{S}} F_{x, p}(\mu) \mu_{p}(d x) \\
& =\sum_{p \in \mathcal{P}} \int_{\mathcal{S}}\left(x A P(A(\mu))-c_{p}(x)\right) \mu_{p}(d x) \\
& =A P(A(\mu)) \sum_{p \in \mathcal{P}} \int_{\mathcal{S}} x \mu_{p}(d x)-\sum_{p \in \mathcal{P}} \int_{\mathcal{S}} c_{p}(x) \mu_{p}(d x) \\
& =\frac{\pi(A(\mu))}{A(\mu)} A(\mu)-\sum_{p \in \mathcal{P}} \int_{\mathcal{S}} c_{p}(x) \mu_{p}(d x) \\
& =\pi(A(\mu))-\sum_{p \in \mathcal{P}} \int_{\mathcal{S}} c_{p}(x) \mu_{p}(d x) .
\end{aligned}
$$

An efficient state in the tragedy of the commons maximizes (30). To characterize such a state,

\footnotetext{
${ }^{20}$ In aggregative potential games, the quasi-potential function is a simpler analogue of the potential function where information about the population state is captured using the scalar $\alpha_{p} \in[\underline{x}, \bar{x}]$. It provides an easily implementable way to maximize the potential function in such a game and thereby characterize Nash equilibrium of the game. Lahkar [15] introduced this notion for finite strategy aggregative potential games. Cheung and Lahkar [5] extended the notion to single population aggregative potential games with continuous strategy sets. The continuous strategy version has been further generalized to multiple populations by Lahkar and Sultana [17]. In terms of this terminology, the function $\bar{G}$ defined by (10) is the quasi-potential function of the potential game $\hat{F}$. It acts as the analogue of the potential function $\bar{F}$ in Theorem 4.2 and Proposition 3.5.

${ }^{21}$ This is assuming that $\alpha_{p}^{*} \in(\underline{x}, \bar{x})$ for all $p \in \mathcal{P}$.
} 
we define the $\bar{G}$ function for this model as

$$
\bar{G}\left(\alpha_{1}, \cdots, \alpha_{n}\right)=\pi\left(\sum_{p} m_{p} \alpha_{p}\right)-\sum_{p \in \mathcal{P}} m_{p} c_{p}\left(\alpha_{p}\right) .
$$

We now obtain the following characterization of the efficient state in the tragedy of the commons. The result follows from Proposition 3.5. Further details are in its proof in Appendix A.4.1 where we verify that the strict concavity of the production function $\pi$ ensures the satisfaction of Assumption 3.3 .

Proposition 5.6 Consider the tragedy of the commons model F defined by (27). This game has a unique efficient state $\mu^{* *}=\left(m_{1} \delta_{\alpha_{1}^{* *}}, \cdots, m_{n} \delta_{\alpha_{n}^{* *}}\right)$, where $\left(\alpha_{1}^{* *}, \cdots, \alpha_{n}^{* *}\right) \in \prod_{p=1}^{n}[\underline{x}, \bar{x}]$ is the unique maximizer of $\bar{G}$ defined in (31).

If the efficient state $\mu^{* *}$ is as described in Proposition 5.6, then $A\left(\mu^{* *}\right)=\sum_{p} m_{p} \alpha_{p}^{* *}$. Hence, from (31), we can characterize the efficient state as $\pi^{\prime}\left(A\left(\mu^{* *}\right)\right)=c_{p}^{\prime}\left(\alpha_{p}^{* *}\right){ }^{22}$ Thus, as expected, the efficient state involves agents equating their marginal cost of their individual strategy with the marginal product of the aggregate strategy. This is in contrast to the Nash equilibrium characterized in Proposition 5.5 where agents equated the average product to marginal cost.

To implement the efficient state, we need to calculate externalities in the tragedy of the commons. We apply Proposition 4.1 for this purpose. The proof is in Appendix A.4.1.

Corollary 5.7 Consider the tragedy of the commons $F$ defined by (27). The total externality imposed by an agent in population $p$ who plays $x \in \mathcal{S}$ at $\mu \in \Delta$ in $F$ is

$$
e_{x, p}(\mu)=x(M P(A(\mu))-A P(A(\mu)))
$$

where $M P(\alpha)=\pi^{\prime}(\alpha)$ is the marginal product at the aggregate strategy level $\alpha$. Moreover, $e_{x, p}(\mu)<$ 0. Therefore, externalities are negative in the tragedy of the commons.

The planner now introduces the variable externality price scheme $t_{x, p}(\mu)=e_{x, p}(\mu)$. As with the public bads model, this transfer is a tax due to externalities being negative. This generates the externality adjusted tragedy of the commons in which, following (14), (27) and (32), takes the form

$$
\begin{aligned}
\hat{F}_{x, p}(\mu) & =F_{x, p}(\mu)+t_{x, p}(\mu) \\
& =x A P(A(\mu))-c_{p}(x)+x(M P(A(\mu))-A P(A(\mu))) \\
& =x M P(A(\mu))-c_{p}(x) .
\end{aligned}
$$

\footnotetext{
${ }^{22}$ As with the Nash equilibrium strategy levels $\alpha_{p}^{*}$ in footnote 21 , here also, we are assuming that the efficient strategy levels $\alpha_{p}^{* *} \in(\underline{x}, \bar{x})$ for all $p$.
} 
As was the case with the externality adjusted public bads game (26), (33) also represents an aggregative game. Therefore, the tragedy of the commons also satisfies Assumption 4.3. This gives us the following result.

Proposition 5.8 Consider the externality adjusted tragedy of the commons $\hat{F}$ defined in (33) obtained through the variable externality pricing scheme $t_{x, p}(\mu)=e_{x, p}(\mu)$, where $e_{x, p}(\mu)$ is as characterized in (32). This game has a unique Nash equilibrium $\mu^{* *}$, which is the unique efficient state of the tragedy of the commons game $F$ as characterized in Proposition 5.6. Further, the replicator dynamic (16), the BNN dynamic (17), the pairwise comparison dynamic (18) and the BR dynamic (21) evolutionarily implement $\mu^{* *}$ as per Theorem 4.4.

Proof. We have verified in the proof of Proposition 5.6 that the tragedy of the commons $F$ satisfies Assumptions 3.1 and 3.3. It also satisfies Assumption 4.3 as is evident from (33). The result, therefore, follows from Theorems 4.2 and 4.4 .

\section{Evolutionary Implementation and Pigouvian Pricing}

The idea of evolutionary implementation clearly derives from the classical notion of Pigouvian pricing. Under both concepts, agents are made to internalize the externality they create, which leads to the realization of the socially optimal outcome. There is, however, one major conceptual difference. Pigouvian pricing is calculated with respect to the socially optimal state. In contrast, evolutionary implementation relies on a variable externality price, i.e. an externality price that varies according to the social state. Nevertheless, as long as the efficient state is uniquely defined, Pigouvian pricing and evolutionary implementation must lead to the same outcome.

To illustrate this claim, let us consider the aggregative game $F$ defined by (7). Proposition 3.5 characterizes its efficient state $\mu^{* *}=\left(m_{1} \delta_{\alpha_{1}^{* *}}, \cdots, m_{n} \delta_{\alpha_{n}^{* *}}\right)$. We can, therefore, use (6) to denote the aggregate strategy level at this state as

$$
\alpha^{* *}=A\left(\mu^{* *}\right)=\sum_{p \in \mathcal{P}} \int_{\mathcal{S}} x \mu_{p}^{* *}(d x)=\sum_{p \in \mathcal{P}} m_{p} \alpha_{p}^{* *}
$$

Inserting (34) into (12), we can then write the total externality imposed by strategy $x$ users in population $p$ at $\mu^{* *}$ as $e_{x, p}\left(\mu^{* *}\right)=x \sum_{q \in \mathcal{P}} \int_{\mathcal{S}} \beta_{q, 2}\left(y, A\left(\mu^{* *}\right)\right) \mu_{q}^{* *}(d y)=x \sum_{q \in \mathcal{P}} \int_{\mathcal{S}} \beta_{q, 2}\left(y, \alpha^{* *}\right) \mu_{q}^{* *}(d y)$. Hence, the Pigouvian price imposed on a strategy $x$ user in population $p$ becomes

$$
t_{x, p}\left(\mu^{* *}\right)=e_{x, p}\left(\mu^{* *}\right)=x \sum_{q \in \mathcal{P}} \int_{\mathcal{S}} \beta_{q, 2}\left(y, \alpha^{* *}\right) \mu_{q}^{* *}(d y) .
$$

This is the same transfer calculated in (13), but specifically with reference to $\mu^{* *}$.

A key informational assumption behind (35) is that the planner who imposes this transfer actually knows the efficient aggregate strategy level $\alpha^{* *}$. We, therefore, make the following assumption about the manner in which the planner implements Pigouvian pricing. 
Assumption 6.1 To implement Pigouvian pricing in the aggregative game $F$ defined by (7), the planner specifies the efficient aggregate strategy level $\alpha^{* *}$ defined by (34) and the Pigouvian price $t_{x, p}\left(\mu^{* *}\right)$ defined by (35).

The justification behind this assumption is that if the planner can calculate the Pigouvian price at $\alpha^{* *}$, there is no additional informational difficulty in specifying that level of aggregate strategy as well.

Once Pigouvian pricing is imposed as per Assumption 6.1, an agent from population $p$ who uses strategy $x$ in the aggregative game $F$ obtains payoff

$$
\pi_{p}(x)=\beta_{p}\left(x, \alpha^{* *}\right)+x \sum_{q \in \mathcal{P}} \int_{\mathcal{S}} \beta_{q, 2}\left(y, \alpha^{* *}\right) \mu_{q}(d y) .
$$

This payoff is the same as in the externality adjusted aggregative game (14) except that it is calculated specifically at $A\left(\mu^{* *}\right)=\alpha^{* *}$. However, unlike (14) which represents a population game, there is no strategic interaction in (36). Instead, it is simply an individual maximization problem. We, therefore, make the reasonable assumption that agents choose their optimal strategy in this problem by maximizing $(36)$ on $\mathcal{S}$. Clearly, the optimal strategy for a population $p$ agent in (36) is then $b_{p}\left(\alpha^{* *}\right)$, which the best response (20) calculated at $\alpha^{* *}$. This leads to the following result on the equivalence of the maximizers of (36) and the function $\bar{G}$ defined by (10). To avoid tedious boundary issues, we assume that (36) and (10) have interior maximizers in this lemma. The proof, which is in Appendix A.5, then follows from the standard first order conditions.

Proposition 6.2 Consider the individual maximization problem $\pi$ as defined in (36). Also consider the function $\bar{G}$ as defined in (10). Note that the optimal strategy for a population $p$ agent in (36) is $b_{p}\left(\alpha^{* *}\right)$, which the best response (20) calculated at $\alpha^{* *}$. Then, $b_{p}\left(\alpha^{* *}\right)=\alpha_{p}^{* *}$, where $\left(\alpha_{1}^{* *}, \cdots, \alpha_{n}^{* *}\right)$ is the unique maximizer of $\bar{G}$ as characterized in Proposition 3.5. Therefore, the social state resulting from individual maximization in $\pi$ is the efficient state $\mu^{* *}=\left(m_{1} \delta_{\alpha_{1}^{* *}}, \cdots, m_{n} \delta_{\alpha_{n}^{* *}}\right)$ of the aggregative game $F$ as characterized in Proposition 3.5.

One notable aspect of this proposition is that the planner only specifies the equilibrium aggregate strategy level $\alpha^{* *}$ while implementing Pigouvian pricing. The type specific strategy levels $\alpha_{p}^{* *}$ are not specified. They arise as a result of individual maximization in $\pi$.

Proposition 6.2, therefore, shows that standard Pigouvian pricing, like evolutionary implementation, also implements the efficient state of $F$. What then is the relevance of evolutionary implementation? The relevance arises from the fact that the informational requirement in evolutionary implementation is significantly less. Pigouvian pricing requires the planner to know optimal aggregate strategy level $\alpha^{* *}$, which cannot be observed unless the society is already at $\mu^{* *}$, which would make the problem redundant. Unless we make the unrealistic assumption that the planner has detailed knowledge of the type distribution and payoff functions (see (10) and Proposition 3.5), it is not possible for the planner to know $\alpha^{* *}$. On the other hand, as discussed at the end of Section 
4, evolutionary implementation depends upon the current externality level $e_{x, p}(\mu)$ which can be empirically estimated. Hence, it may be a more realistic way to solve implementation problems. Of course, unlike Pigouvian pricing, it does not ensure instantaneous coordination on the efficient outcome. Instead, it is a slower approach in which the variable price needs to be constantly updated. But it can succeed in its objective by nudging the society towards the efficient state through repeated updating.

\section{Conclusion}

This paper has considered evolutionary implementation in a general environment of aggregative games. Due to the presence of externalities, there is a divergence between Nash equilibrium and the efficient state in such games, where the efficient state is the state that maximizes aggregate payoff. The solution to the problem is to impose a variable externality pricing scheme which equals the externality created by agents. Agents are, therefore, compelled to internalize the externality they create. The resulting externality adjusted game is a potential game with the aggregate payoff function of the original game being its potential function. The maximizer of this function is, therefore, the Nash equilibrium of the externality adjusted game to which standard evolutionary dynamics converge. Since this maximizer is also the efficient state of the original game, we obtain evolutionary implementation.

Our interest in aggregative games is for two reasons. First, our earlier application to the public goods model suggested that aggregative games have certain features that allow for a parsimonious characterization of efficiency. This paper shows that is indeed the case. If the payoff function of the game satisfies certain concavity assumptions, then the game does have a unique efficient state that can be easily calculated. Second, apart from the public goods model, other important economic situations can also be modeled as aggregative games. In this paper, we consider two such applications - public bads and tragedy of the commons. Both represent a wide variety of social problems and our analysis provides a general framework to understand evolutionary implementation in such situations. The important feature of evolutionary implementation is that it allows the planner to nudge the society to the efficient state by imposing transfers based on the current level of externality, which can be empirically estimated. This may not be the case, for example, with classical Pigouvian pricing which requires the planner to know the efficient state a priori.

We conclude with a discussion of extending the large population implementation approach to general equilibrium settings. Consider a situation where agents have (possibly heterogenous) preferences over resources and each agent is endowed with some resources. Suppose that the agents are strategic with respect to reporting their endowments. Prices in the market are determined by the aggregate endowment reported. The resource allocation in the Nash equilibrium in such a setting may not be Pareto efficient without a planner's intervention. In order to implement a Pareto efficient allocation, the planner needs to acquire information about the preferences and endowments of the agents. Thus in a setting where such information is private, the allocation rule has to be 
incentive compatible or strategy-proof. The literature on strategy-proof allocation rules in general economic environments mostly consists of impossibility results. For example, Theorem 4.4.1 in Dasgupta et al. [8] shows that only Pareto efficient and strategy-proof rules in such a setting is the dictatorial rule.

Large economies are, however, special in this regard. Hurwicz [13] observes that in a large economy, no agent has any influence on determining the price by reporting a false preference and hence the competitive mechanism ${ }^{23}$ is strategy-proof in a large economy. Hammond [10] is another important paper in this regard. It shows that in a large exchange economy with suitable assumptions on the feasible consumption sets and types ${ }^{24}$, an incentive compatible competitive mechanism exists and the mechanism requires no lump-sum transfer. In fact, Theorem 5 in Hammond [10] says that the Pareto efficient and incentive compatible allocations result in only if the lump sum transfers are zero. It would be interesting to review these results in our setting and explore the connection of the variable externality pricing in our mechanism with the transfer function as discussed in Hammond $[10]$.

\section{A Appendix}

\section{A.1 Proofs in Section 2}

We define the Fréchet derivative as follows.

Definition A.1 Let $X$ and $Y$ are Banach spaces. We say that $g: X \rightarrow Y$ is Fréchet-differentiable at $x$ if there exists a continuous linear map $T: X \rightarrow Y$ such that $g(x+\vartheta)=g(x)+T \vartheta+o(\|\vartheta\|)$ for all $\vartheta$ in some neighborhood of zero in $X$. If it exists, this $T$ is called the Fréchet-derivative of $g$ at $x$, and is written as $D g(x)$.

To apply the Fréchet derivative in our context, we need to define the variatonal norm on $\mathcal{M}$. First, we impose this norm on $\mathcal{S}$. For $\nu \in \mathcal{M}(\mathcal{S})$, the variational norm is given by $\|\nu\|=$ $\sup _{g}\left|\int_{\mathcal{S}} g d \nu\right|$ where $g$ is a measurable function $g: \mathcal{S} \rightarrow \mathbf{R}$ such that $\sup _{x \in S}|g(x)| \leq 1$. The variational norm on $\mathcal{M}=\prod_{p \in \mathcal{P}} \mathcal{M}(\mathcal{S})$ is given by (see, for example, Perkins and Leslie [26])

$$
\|\mu\|=\max \left\{\left\|\mu_{1}\right\|, \cdots,\left\|\mu_{n}\right\|\right\} \text { for } \mu=\left(\mu_{1}, \cdots, \mu_{n}\right) \in \mathcal{M} \text {. }
$$

With this norm, we obtain the Banach space $(\mathcal{M},\|\cdot\|)$ on which we can calculate Fréchet derivatives. This leads to the following proof.

Proof of Proposition 2.4: First, we extend the domain of $\bar{F}$ from $\Delta$ to $\mathcal{M}$. This implies that for every $(x, p) \in \mathcal{S} \times \mathcal{P}$, the domain of $F_{x, p}(\mu)$ also extends from $\Delta$ to $\mathcal{M}$. Definition 2.3 requires

\footnotetext{
${ }^{23}$ We call a mechanism "competitive" if it implements the competitive equilibrium at any state.

${ }^{24}$ In Hammond citeHammond1979 type of an agent consists of information on both her endowment and preferences.
} 
us to show that

$$
\nabla \bar{F}(\mu)(x, p)=\hat{F}_{x, p}(\mu)=F_{x, p}(\mu)+\sum_{q \in \mathcal{P}} \int_{\mathcal{S}} D F_{y, q}(\mu) \mathcal{D}_{x, p} \mu_{q}(d y) .
$$

Consider $\zeta=\left(\zeta_{1}, \cdots, \zeta_{n}\right) \in \mathcal{M}$. Note that

$$
F_{x, p}(\mu+\zeta)=F_{x, p}(\mu)+D F_{x, p}(\mu) \zeta+o(\|\zeta\|),
$$

where $\|\cdot\|$ is the variational norm on $\mathcal{M}$ as defined in (37). Hence,

$$
\begin{aligned}
\bar{F}(\mu+\zeta) & =\sum_{q \in \mathcal{P}} \int_{\mathcal{S}} F_{y, q}(\mu+\zeta)\left(\mu_{q}+\zeta_{q}\right)(d y) \\
& =\sum_{q \in \mathcal{P}} \int_{\mathcal{S}}\left(F_{y, q}(\mu)+D F_{y, q}(\mu) \zeta+o(\|\zeta\|)\right)\left(\mu_{q}+\zeta_{q}\right)(d y)(\text { from }(39)) \\
& =\sum_{q \in \mathcal{P}} \int_{\mathcal{S}} F_{y, q}(\mu) \mu_{q}(d y)+\sum_{q \in \mathcal{P}} \int_{\mathcal{S}} F_{y, q}(\mu) \zeta_{q}(d y)+\sum_{q \in \mathcal{P}} \int_{\mathcal{S}} D F_{y, q}(\mu) \zeta \mu_{q}(d y)+o(\|\zeta\|) \\
& =\bar{F}(\mu)+\sum_{q \in \mathcal{P}} \int_{\mathcal{S}} F_{y, q}(\mu) \zeta_{q}(d y)+\sum_{q \in \mathcal{P}} \int_{\mathcal{S}} D F_{y, q}(\mu) \zeta \mu_{q}(d y)+o(\|\zeta\|)
\end{aligned}
$$

Applying Definition A.1 to (40), we obtain

$$
D \bar{F}(\mu) \zeta=\sum_{q \in \mathcal{P}} \int_{\mathcal{S}} F_{y, q}(\mu) \zeta_{q}(d y)+\sum_{q \in \mathcal{P}} \int_{\mathcal{S}} D F_{y, q}(\mu) \zeta \mu_{q}(d y)
$$

The definition of the gradient in (5) then implies that

$$
\sum_{q} \int_{\mathcal{S}} \nabla \bar{F}(\mu)(y, q) \zeta_{q}(d y)=\sum_{q \in \mathcal{P}} \int_{\mathcal{S}} F_{y, q}(\mu) \zeta_{q}(d y)+\sum_{q \in \mathcal{P}} \int_{\mathcal{S}} D F_{y, q}(\mu) \zeta \mu_{q}(d y) .
$$

This equation holds for all $\zeta \in \mathcal{M}$. Hence, it holds for

$$
\zeta=\mathcal{D}_{x, p} \text { in which } \zeta_{p}=\delta_{x} \text { and } \zeta_{q}=0 \text { for all } q \in \mathcal{P} \backslash p \text { (see (3)). }
$$

Applying $\mathcal{D}_{x, p}$ to (41), we obtain (38).

\section{A.2 Proofs of Section 3}

Proof of Lemma 3.4: For the concavity part, consider $\mu, \nu \in \Delta$. note from (6) that $A(\cdot)$ is linear. Hence, if $A(\mu)=A(\nu)$, then

$$
A(\lambda \mu+(1-\lambda) \nu)=\lambda A(\mu)+(1-\lambda) A(\nu)=A(\mu)=A(\nu)
$$


In that case, the two sides of (9) must be equal. This rules out strict concavity. But due to Assumption 3.3, (9) must hold if $A(\mu) \neq A(\nu)$. This implies $\bar{F}(\lambda \mu+(1-\lambda) \nu) \geq \lambda \bar{F}(\mu)+(1-\lambda) \bar{F}(\nu)$, with equality holding only for such $\mu, \nu$ such that $A(\mu)=A(\nu)$. Hence, $\bar{F}$ is concave but not strictly concave.

For the second part of the lemma, suppose $\mu_{q}^{* *}$ is not monomorphic for some $q \in \mathcal{P}$. Define $a\left(\mu_{q}\right)=\int_{\mathcal{S}} x \mu_{q}(d x)$ as the aggregate strategy level in population $q$. Let $a\left(\mu_{q}^{* *}\right)=\alpha_{q} \in\left[0, m_{q} \bar{x}\right]$. Consider a new social state $\hat{\mu}$ such that $\hat{\mu}_{p}=\mu_{p}^{* *}$ for all $p \neq q$ and $\hat{\mu}_{q}=m_{q} \delta \frac{\alpha_{q}}{m_{q}}$. Thus, in constructing $\hat{\mu}$, we have replaced the non-monomorphic population state $\mu_{q}^{* *}$ with a monomorphic state with the same population level aggregate strategy.

Clearly, $A\left(\mu^{* *}\right)=A(\hat{\mu})$ and $\int_{\mathcal{S}} \beta_{p}\left(x, A\left(\mu^{* *}\right)\right) \mu_{p}^{* *}(d x)=\int_{\mathcal{S}} \beta_{p}(x, A(\hat{\mu})) \hat{\mu}_{p}(d x)$ for all $p \neq q$. But since $\mu_{q}^{* *}$ is not monomorphic, the strict concavity of $\beta_{q}$ with respect to $x$ (Assumption 3.1) implies that

$$
\begin{aligned}
& \beta_{q}\left(\int_{\mathcal{S}} x \frac{\mu_{q}^{* *}}{m_{q}}(d x), A\left(\mu^{* *}\right)\right)>\int_{\mathcal{S}} \beta_{q}\left(x, A\left(\mu^{* *}\right)\right) \frac{\mu_{q}^{* *}}{m_{q}}(d x) \\
\Rightarrow & m_{q} \beta_{q}\left(\frac{a\left(\mu_{q}^{* *}\right)}{m_{q}}, A\left(\mu^{* *}\right)\right)>\int_{\mathcal{S}} \beta_{q}\left(x, A\left(\mu^{* *}\right)\right) \mu_{q}^{* *}(d x) \\
\Rightarrow & m_{q} \beta_{q}\left(\frac{\alpha_{q}}{m_{q}}, A\left(\mu^{* *}\right)\right)>\int_{\mathcal{S}} \beta_{q}\left(x, A\left(\mu^{* *}\right)\right) \mu_{q}^{* *}(d x) \\
\Rightarrow & \int_{\mathcal{S}} \beta_{q}\left(x, A\left(\mu^{* *}\right)\right) \hat{\mu}_{q}(d x)>\int_{\mathcal{S}} \beta_{q}\left(x, A\left(\mu^{* *}\right)\right) \mu_{q}^{* *}(d x) \\
\Rightarrow & \int_{\mathcal{S}} \beta_{q}(x, A(\hat{\mu})) \hat{\mu}_{q}(d x)>\int_{\mathcal{S}} \beta_{q}\left(x, A\left(\mu^{* *}\right)\right) \mu_{q}^{* *}(d x),
\end{aligned}
$$

where the left hand sides of (43) and (44) arise because $A\left(\mu^{* *}\right)=A(\hat{\mu})$. Since we have already argued that $\int_{\mathcal{S}} \beta_{p}\left(x, A\left(\mu^{* *}\right)\right) \mu_{p}^{* *}(d x)=\int_{\mathcal{S}} \beta_{p}(x, A(\hat{\mu})) \hat{\mu}_{p}(d x)$ for all $p \neq q$, (44) implies that $\mu^{* *}$ in which any population state is not monomorphic could not have been a maximizer of $\bar{F}(\mu)=$ $\sum_{p} \int_{\mathcal{S}} \beta_{p}(x, A(\mu)) \mu_{p}(d x)$.

To show uniqueness, suppose $\hat{\mu}$ and $\hat{\nu}$ are two maximizers of $\bar{F}$. The above argument establishes that both $\hat{\mu}$ and $\hat{\nu}$ are in monomorphic population states. Since $\bar{F}$ is concave, it has a convex set of maximizers. Hence, $\frac{1}{2} \hat{\mu}+\frac{1}{2} \hat{\nu}$ must also be maximizer of $\bar{\mu}$. But unless $\hat{\mu}=\hat{\nu}$, there must exist at least one population $p$ such that $\frac{1}{2} \hat{\mu}_{p}+\frac{1}{2} \hat{\nu}_{p}$ is not monomorphic. But this is a contradiction. Hence, $\bar{F}$ cannot have more than one maximizer.

Proof of Proposition 3.5: Assumptions 3.1 and 3.3 hold. Hence, by Lemma 3.4, $F$ has a unique efficient state in monomorphic population states.

To characterize the maximizer of $\bar{G}$, we first show that it is strictly concave on $\prod_{p=1}^{n}[\underline{x}, \bar{x}] \rightarrow \mathbf{R}$. Consider two points $\left(\hat{\alpha}_{1}, \cdots, \hat{\alpha}_{n}\right) \neq\left(\tilde{\alpha}_{1}, \cdots, \tilde{\alpha}_{n}\right) \in \prod_{p=1}^{n}[\underline{x}, \bar{x}]$ and define $\hat{\mu}=\left(m_{1} \delta_{\hat{\alpha}_{1}}, \cdots, m_{1} \delta_{\hat{\alpha}_{n}}\right) \in$ $\Delta$ and $\tilde{\mu}=\left(m_{1} \delta_{\tilde{\alpha}_{1}}, \cdots, m_{1} \delta_{\tilde{\alpha}_{n}}\right) \in \Delta$. Clearly, $\bar{G}\left(\hat{\alpha}_{1}, \cdots, \hat{\alpha}_{n}\right)=\bar{F}(\hat{\mu})$ and $\bar{G}\left(\tilde{\alpha}_{1}, \cdots, \tilde{\alpha}_{n}\right)=\bar{F}(\tilde{\mu})$. Further, $A(\hat{\mu})=\sum_{q} m_{q} \hat{\alpha}_{q}$ and $A(\tilde{\mu})=\sum_{q} m_{q} \tilde{\alpha}_{q}$.

We now need to examine two case. Suppose first that $\sum_{q} m_{q} \hat{\alpha}_{q} \neq \sum_{q} m_{q} \tilde{\alpha}_{q}$. Then, due to the 
above characteristics of $\bar{F}, \bar{G}$ and $A(\cdot),(9)$ in Assumption 3.3 implies that

$$
\begin{aligned}
\sum_{p} m_{p} \beta_{p}\left(\lambda \hat{\alpha}_{p}+(1-\lambda) \tilde{\alpha}_{p}, \sum_{q} m_{q}\left(\lambda \hat{\alpha}_{q}+(1-\lambda) \tilde{\alpha}_{q}\right)\right) & >\lambda \sum_{p} m_{p} \beta_{p}\left(\hat{\alpha}_{p}, \sum_{q} m_{q} \hat{\alpha}_{q}\right) \\
& +(1-\lambda) \sum_{p} m_{p} \beta_{p}\left(\tilde{\alpha}_{p}, \sum_{q} m_{q} \tilde{\alpha}_{q}\right)
\end{aligned}
$$

which, by the definition of $\bar{G}$, is equivalent to

$$
\bar{G}\left(\lambda \hat{\alpha}_{1}+(1-\lambda) \tilde{\alpha}_{1}, \cdots, \lambda \hat{\alpha}_{n}+(1-\lambda) \tilde{\alpha}_{n}\right)>\lambda \bar{G}\left(\hat{\alpha}_{1}, \cdots, \hat{\alpha}_{n}\right)+(1-\lambda) \bar{G}\left(\tilde{\alpha}_{1}, \cdots, \tilde{\alpha}_{n}\right) .
$$

Second, suppose $\sum_{q} m_{q} \hat{\alpha}_{q}=\sum_{q} m_{q} \tilde{\alpha}_{q}$. Then, $\sum_{q} m_{q}\left(\lambda \hat{\alpha}_{q}+(1-\lambda) \tilde{\alpha}_{q}\right)=\sum_{q} m_{q} \hat{\alpha}_{q}=\sum_{q} m_{q} \tilde{\alpha}_{q}$. However, for at least one $k \in \mathcal{P}, \hat{\alpha}_{k} \neq \tilde{\alpha}_{k}$. Assumption 3.1 then implies that for every $p$,

$$
\begin{aligned}
\beta_{k}\left(\lambda \hat{\alpha}_{k}+(1-\lambda) \tilde{\alpha}_{k}, \sum_{q} m_{q}\left(\lambda \hat{\alpha}_{q}+(1-\lambda) \tilde{\alpha}_{q}\right)\right) & \geq \lambda \beta_{k}\left(\hat{\alpha}_{k}, \sum_{q} m_{q} \hat{\alpha}_{q}\right) \\
& +(1-\lambda) \beta_{k}\left(\tilde{\alpha}_{k}, \sum_{q} m_{q} \tilde{\alpha}_{q}\right)
\end{aligned}
$$

with the inequality being strict for any $k \in \mathcal{P}$ such that $\hat{\alpha}_{k} \neq \tilde{\alpha}_{k}$. But this means that even if $\sum_{q} m_{q} \hat{\alpha}_{q}=\sum_{q} m_{q} \tilde{\alpha}_{q}$, (45) and, hence, (46) holds.

These two cases suffice to establish the strict concavity of $\bar{G}$. Hence, $\bar{G}$ has a unique maximizer in $\prod_{p=1}^{n}[\underline{x}, \bar{x}]$, which we denote $\left(\alpha_{1}^{* *}, \cdots, \alpha_{n}^{* *}\right)$. We know that $\bar{F}(\mu)=\bar{G}\left(\alpha_{1}, \cdots, \alpha_{n}\right)$ if $\mu=\left(m_{1} \delta_{\alpha_{1}}, \cdots, m_{n} \delta_{\alpha_{n}}\right)$. Moreover, Lemma 3.4 implies that the efficient state of $F$ must be in monomorphic population states. Together, these arguments imply that the efficient state of $F$ is $\mu^{* *}=\left(m_{1} \delta_{\alpha_{1}^{* *}}, \cdots, m_{n} \delta_{\alpha_{n}^{* *}}\right)$.

\section{A.3 Proofs in Section 4}

Proof of Proposition 4.1: We apply (3). Consider $\zeta=\left(\zeta_{1}, \cdots, \zeta_{n}\right)$. Note that $F_{y, q}(\mu)=$ $\beta_{q}(y, A(\mu))$. We apply the chain rule for the Fréchet derivative to obtain

$$
\begin{aligned}
D F_{y, q}(\mu) \zeta & =\beta_{q, 2}(y, A(\mu)) D A(\mu) \zeta \\
& =\beta_{q, 2}(y, A(\mu)) \int_{\mathcal{S}} \tilde{x} \sum_{k \in \mathcal{P}} \zeta_{k}(d \tilde{x}) .
\end{aligned}
$$

Using $\zeta=\mathcal{D}_{x, p}($ see $(42))$ in $(48)$, we obtain

$$
D F_{y, q}(\mu) \mathcal{D}_{x, p}=x \beta_{q, 2}(y, A(\mu))
$$


This is the externality imposed by strategy $-x$ agents in population $p$ on strategy $-y$ agents in population $q$. Using (3), we then obtain the total externality imposed by strategy- $x$ agents in population $p$ on society to be

$$
e_{x, p}(\mu)=\sum_{q \in \mathcal{P}} \int_{\mathcal{S}} D F_{y, q}(\mu) \mathcal{D}_{x, p} \mu_{q}(d y)=x \sum_{q \in \mathcal{P}} \int_{\mathcal{S}} \beta_{q, 2}(y, A(\mu)) \mu_{q}(d y) .
$$

\section{A.4 Proofs in Section 5}

Proof of Proposition 5.1: We first check whether $F$ satisfies Assumptions 3.1 and 3.3. Then, the result would follow from Proposition 3.5.

Assumption 3.1 follows readily from the fact that $v_{p}$ is strictly concave in (22). For Assumption 3.3 , note that

$$
\sum_{p} \int_{\mathcal{S}} \beta_{p}(x, A(\mu)) \mu_{p}(d x)=\sum_{p} \int_{\mathcal{S}} v_{p}(x) \mu_{p}(d x)-\sum_{p} m_{p} c_{p}(A(\mu))
$$

This is, of course, the right hand side of (23). Here, $\sum_{p} \int_{\mathcal{S}} v_{p}(x) \mu_{p}(d x)$ is linear in $\mu$. Therefore, the satisfaction of (9) and, hence, Assumption 3.3 depends upon the convexity of $\sum_{p} m_{p} c_{p}(A(\mu))$. But this follows from our assumption in (22) that $c_{p}$ is strictly convex in $\alpha \in[\underline{x}, \bar{x}]$. Hence, if $\mu, \nu \in \Delta$ are such that $A(\mu) \neq A(\nu)$, then the strict convexity of $c_{p}$ with respect to $\alpha \in[\underline{x}, \bar{x}]$ ensures $(9)$.

Since both Assumptions 3.1 and 3.3 are satisfied, Proposition 3.5 holds. This implies our desired conclusion.

\section{A.4.1 Tragedy of the Commons}

Consider the general set-up described in Section 2 with strategy set $\mathcal{S}$, set of social states $\Delta$ and set of populations $\mathcal{P}$. Lahkar and Sultana [17] consider games in which the payoff to an agent in population $p$ who plays strategy $x$ at social state $\mu$ is

$$
F_{x, p}(\mu)=\phi_{p}(x) \beta(A(\mu))-c_{p}(x)
$$

in which $A(\mu)=\sum_{p} \int_{\mathcal{S}} \phi_{p}(x) \mu_{p}(d x)$ and where $\beta:[\underline{x}, \bar{x}] \rightarrow \mathbf{R}_{+}$is strictly declining, $\phi_{p}: \mathcal{S} \rightarrow \mathbf{R}_{+}$is concave and $c_{p}: \mathcal{S} \rightarrow \mathbf{R}$ is convex, with the further restriction that if $\phi_{p}$ is linear, then $c_{p}$ is strictly convex. The tragedy of the commons defined by (27) is clearly an example of (52) with $\phi_{p}(x)=x$ for all $p$ and $\beta(A(\mu))=A P(A(\mu))$. Lahkar and Sultana [17] call games of the form (52) generalized aggregative games and show that they constitute potential games with potential functions of the form

$$
f(\mu)=\int_{\underline{x}}^{A(\mu)} \beta(z)-\sum_{p} \int_{\mathcal{S}} c_{p}(x) \mu_{p}(d x)
$$


where $A(\mu)=\sum_{p} \int_{\mathcal{S}} \phi_{p}(x) \mu_{p}(d x)$. They show that (53) is a concave, but not strictly concave, function in $\Delta$ and, therefore, has a convex set of maximizers. This leads to the following proof of Proposition 5.4.

Proof of Proposition 5.4: The fact that the tragedy of the commons $F$ is a potential game with potential function $f$ defined by (28) follows from Proposition 4.2 in Lahkar and Sultana [17]. The concavity, but not strict concavity, of the potential function follows from Lemma 4.3 in Lahkar and Sultana [17].

We provide a brief sketch of the details of the above proof. To show that $f$ is the potential function, we need to apply the Fréchet derivative and show that

$$
\nabla f(\mu)(x, p)=F_{x, p}(\mu)=x A P(A(\mu))-c_{p}(x)
$$

This follows because the Fréchet derivative of $f(\mu)$ in the direction $\zeta$ is $D f(\mu) \zeta=\beta(A(\mu)) D A(\mu) \zeta-$ $D C(\mu) \zeta$, where $C(\mu)=\sum_{p} \int_{\mathcal{S}} c_{p}(x) \mu_{p}(d x)$ is the aggregate cost at $\mu$. Replacing $\zeta$ with the measure $\mathcal{D}_{x, p}$ defined in (3) and using Definition 2.3 gives us (54).

For concavity of $f$, note that $C(\mu)=\sum_{p} \int_{\mathcal{S}} c_{p}(x) \mu_{p}(d x)$ is linear in $\mu$. On the other hand, because $A P(z)$, by assumption, is strictly declining, $\int_{\underline{x}}^{A}(\mu) A P(z) d z$ is concave. Strict concavity is ruled out because it is possible that $A(\mu)=A(\nu)$ even if $\mu \neq \nu$.

In order to maximize the potential function (53), Lahkar and Sultana [17] define its quasipotential function $g: \prod_{p}[\underline{x}, \bar{x}] \rightarrow \mathbf{R}$ as (see also footnote 20 )

$$
g\left(\alpha_{1}, \alpha_{2}, \cdots, \alpha_{n}\right)=\int_{\sum_{p \in \mathcal{P}} m_{p} \phi_{p}(\underline{x})}^{\sum_{p \in \mathcal{P}} m_{p} \phi_{p}\left(\alpha_{p}\right)} \beta(z) d z-\sum_{p \in \mathcal{P}} m_{p} c_{p}\left(\alpha_{p}\right) .
$$

They show that (55) is a strictly convex function with a unique maximizer $\left(\alpha_{1}^{* *}, \cdots, \alpha_{n}^{* *}\right) \in$ $\prod_{p=1}^{n}[\underline{x}, \bar{x}]$. They then show that the social state $\mu^{*}=\left(m_{1} \delta_{\alpha_{1}^{*}}, m_{2} \delta_{\alpha_{2}^{*}}, \cdots, m_{n} \delta_{\alpha_{n}^{*}}\right)$ is the unique maximizer of the potential function (53). Since the potential function is concave, $\mu^{*}$ must also be the unique Nash equilibrium of $F$ defined by (52). We, therefore, obtain the following proof of Proposition 5.5.

Proof of Proposition 5.5: Follows from Theorem 5.3 in Lahkar and Sultana [17].

We provide a sketch of the arguments that allow us to apply Lahkar and Sultana's [17] to the tragedy of the commons. Consider $f$ as defined in (28) and $g$ as defined in (29). First, note that if $\mu$ is in monomorphic population states of the form $\mu_{p}=m_{p} \delta_{\alpha_{p}}$, then $g\left(\alpha_{1}, \cdots, \alpha_{n}\right)=f(\mu)$. On the other hand, if $\mu_{p}$ is polymorphic for any $p$, then define $a\left(\mu_{p}\right)=\int_{\mathcal{S}} x \mu_{p}(d x)$. Then, due to the strict convexity $c_{p}, g\left(\alpha_{1}, \cdots, \alpha_{n}\right)>f(\mu)$. Further details of this argument are in Lemma 5.1 of Lahkar and Sultana [17].

Next, we establish the strict convexity of $g$. Since $A P(z)$ is strictly declining, $\int_{\underline{x}}^{\sum_{p \in \mathcal{P}} m_{p} \alpha_{p}} A P(z) d z$ 
is concave on $\prod_{p}[\underline{x}, \bar{x}]$. Strict concavity is ruled out because it may be that $\sum_{p \in \mathcal{P}} m_{p} \hat{\alpha}_{p}=$ $\sum_{p \in \mathcal{P}} m_{p} \tilde{\alpha}_{p}$ even if $\left(\hat{\alpha}_{1}, \cdots, \hat{\alpha}_{n}\right) \neq\left(\tilde{\alpha}_{1}, \cdots, \tilde{\alpha}_{n}\right)$. On the other hand, $\sum_{p} m_{p} c_{p}\left(\alpha_{p}\right)$ is strictly convex due to the strict convexity of $c_{p}$. Hence, $g$ is strictly convex. Hence, $g$ has a unique maximizer, which we denote $\left(\alpha_{1}^{*}, \cdots, \alpha_{n}^{*}\right)$. Further details are in Lemma 5.2 of Lahkar and Sultana $[17]$.

Now consider $\mu^{*}=\left(m_{1} \delta_{\alpha_{1}^{*}}, \cdots, m_{n} \delta_{\alpha_{n}^{*}}\right)$. Since this is entirely in monomorphic population states, our earlier argument establishes that $f\left(\mu^{*}\right)=g\left(\alpha_{1}^{*}, \cdots, \alpha_{n}^{*}\right)$. Consider $\mu \neq \mu^{*}$. If $\mu$ is in monomorphic population states of the form $\mu_{p}=m_{p} \delta_{\alpha_{p}}$, then $\mu \neq \mu^{*}$ implies $\left(\alpha_{1}^{*}, \cdots, \alpha_{n}^{*}\right) \neq$ $\left(\alpha_{1}, \cdots, \alpha_{n}\right)$. Hence, $f\left(\mu^{*}\right)=g\left(\alpha_{1}^{*}, \cdots, \alpha_{n}^{*}\right)>g\left(\alpha_{1}, \cdots, \alpha_{n}\right)=f(\mu)$. On the other hand, if $\mu_{p}$ is polymorphic for some $p$, define $\alpha_{p}=\frac{a\left(\mu_{p}\right)}{m_{p}}$. Then, $f\left(\mu^{*}\right)=g\left(\alpha_{1}^{*}, \cdots, \alpha_{n}^{*}\right) \geq g\left(\alpha_{1}, \cdots, \alpha_{n}\right)>f(\mu)$. Therefore, for any $\mu \neq \mu^{*}, f\left(\mu^{*}\right)>f(\mu)$. This establishes the desired conclusion in Proposition 5.5. Further details are in Theorem 5.3 in Lahkar and Sultana [17].

Proof of Proposition 5.6: We need to check whether $F$ satisfies Assumptions 3.1 and 3.3. The result then follows from Proposition 3.5.

Assumption 3.1 is satisfied because $c_{p}$ is strictly convex in (27). For Assumption 3.3, note that

$$
\sum_{p} \int_{\mathcal{S}} \beta_{p}(x, A(\mu)) \mu_{p}(d x)=\pi(A(\mu))-\sum_{p} \int_{\mathcal{S}} c_{p}(x) \mu_{p}(d x)
$$

which is the right hand side of $(30)$. Here, $\sum_{p} \int_{\mathcal{S}} c_{p}(x) \mu_{p}(d x)$ is linear in $\mu$. Therefore, the satisfaction Assumption 3.3 depends upon the concavity of $\pi(A(\mu))$. But we have assumed that $\pi$ is strictly concave on $[\underline{x}, \bar{x}]$. Hence, if $\mu, \nu \in \Delta$ are such that $A(\mu) \neq A(\nu)$, then (9) holds.

Since both Assumptions 3.1 and 3.3 are satisfied, Proposition 3.5 holds. This implies our desired conclusion.

Proof of Corollary 5.7: The tragedy of the commons is characterized by $\beta_{q}(y, \alpha)=y A P(\alpha)-$ $c_{p}(y)=y \frac{\pi(\alpha)}{\alpha}-c_{p}(y)$. Hence,

$$
\begin{aligned}
\beta_{q, 2}(y, \alpha) & =y \frac{\alpha \pi^{\prime}(\alpha)-\pi(\alpha)}{\alpha^{2}} \\
& =\frac{y}{\alpha}(M P(\alpha)-A P(\alpha)) .
\end{aligned}
$$

Applying (57) to (12) in Proposition 4.1, we obtain

$$
\begin{aligned}
e_{x, p}(\mu) & =x \frac{M P(A(\mu))-A P(A(\mu))}{A(\mu)} \sum_{p \in \mathcal{P}} \int_{\mathcal{S}} y \mu_{p}(d y) \\
& =x(M P(A(\mu))-A P(A(\mu))) .
\end{aligned}
$$

Since, by assumption, $A P(A(\mu))$ is strictly declining, $M P(A(\mu))<A P(A(\mu))$. Therefore, $e_{x, p}(\mu)<$ 0 . 


\section{A.5 Proofs in Section 6}

Proof of Proposition 6.2: The FOC for maximizing (36) is (assuming an interior maximizer)

$$
\beta_{p 1}\left(b_{p}\left(\alpha^{* *}\right), \alpha^{* *}\right)=-\sum_{q \in \mathcal{P}} \beta_{q 2}\left(b_{q}\left(\alpha^{* *}\right), \alpha^{* *}\right)
$$

where $\alpha^{* *}=\sum_{q} m_{q} \alpha_{q}^{* *}$ as defined in (34). On the other hand, from (10), we obtain the FOC characterizing $\left(\alpha_{1}^{* *}, \cdots, \alpha_{n}^{* *}\right)$ (assuming an interior maximizer) as

$$
\beta_{p 1}\left(\alpha_{p}^{* *}, \alpha^{* *}\right)=-\sum_{q \in \mathcal{P}} \beta_{q 2}\left(\alpha_{q}^{* *}, \alpha^{* *}\right)
$$

where $\alpha^{* *}=\sum_{q} m_{q} \alpha_{q}^{* *}$. But we know from Proposition 3.5 that $\left(\alpha_{1}^{* *}, \cdots, \alpha_{n}^{* *}\right)$ is the unique maximizer of $\bar{G}$. Hence, this must be the unique solution to (59) subject to the condition $\alpha^{* *}=$ $\sum_{q} m_{q} \alpha_{q}^{* *}$. Since this condition also holds for (58), a comparison of (58) and (59) implies that $b_{p}\left(\alpha^{* *}\right)=\alpha_{p}^{* *}$. Therefore, the social state resulting from individual maximization in $(36)$ is $\mu^{* *}$.

\section{References}

[1] Ayres I., Levitt S. D (1998) Measuring positive externalities from unobservable victim precaution: An empirical analysis of LoJack. Quarterly Journal of Economics 113:43--77.

[2] Chay K, Greenstone M (2005) Does air quality matter? Evidence from the housing market. J. Political Econ. 113:376-424.

[3] Cheung MW (2014) Pairwise comparison dynamics for games with continuous strategy space. J. Econ. Theory 153:344-375.

[4] Cheung MW (2016) Imitative Dynamics for Games with Continuous Strategy Space. Games Econ. Behav. 99:206-223.

[5] Cheung MW, Lahkar R (2018) Nonatomic Potential Games: the Continuous Strategy Case. Games Econ. Behav. 108:341-362.

[6] Clarke E (1971) Multi-part pricing of public goods. Public Choice 11:17-23.

[7] Corchón L (1994) Comparative statics for aggregative games the strong concavity case. Math. Soc. Sci. 28:151-165.

[8] Dasgupta P, Hammond P, Maskin E (1979) The implementation of social choice rules: some general results on incentive compatibility. Rev. Econ. Studies. 46: 185-216.

[9] Groves T (1973) Incentives in teams. Econometrica 41:617-631. 
[10] Hammond P (1979) Straightforward individual incentive compatibility in large economies. Rev. Econ. Studies. 46: 263-282.

[11] Hanemann WM (1994) Valuing the environment through contingent valuation, J. Econ. Perspect. 8:19-43.

[12] Hofbauer J, Oechssler J, Riedel F (2009) Brown-von Neumann-Nash dynamics: the continuous strategy case. Games Econ. Behav. 65:406-429.

[13] Hurwicz L (1972) Informationally Decentralized Systems, ch. 14, pp. 297-336 of McGuire, C. B. and Radner, R. (eds.) Decision and Organization (North-Holland).

[14] Lahkar R, Riedel F (2015) The logit dynamic for games with continuous strategy sets. Games Econ. Behav. 91:268-282.

[15] Lahkar R (2017) Large population aggregative potential games. Dyn Games Appl. 7:443-467.

[16] Lahkar R, Mukherkee S (2019) Evolutionary Implementation in a Public Goods Game. J. Econ. Theory 181:423-460.

[17] Lahkar R, Sultana R (2020) Potential Games, Large Population Contests and Affirmative Action. working paper, https://sites.google.com/site/rlahkar/home.

[18] Le Goffe P (2000) Hedonic pricing of agriculture and forestry externalities. Env. and Resource Econ. 15: 397-401.

[19] Li M, Brown H (1980) Micro-neighborhood externalities and Hedonic housing prices. Land Economics 56:125-141.

[20] Lin S (Ed.) (1976) Theory and measurement of economic externalities. Academic Press.

[21] Maskin E, Sjöström T (2002). Implementation Theory. In: Arrow K, Sen A, Suzumura K Handbook of Social Choice Theory Vol. I. Amsterdam: North Holland, pp.237-288.

[22] Monderer D, Shapley L (1996) Potential games. Games Econ. Behav. 14:124-143.

[23] Oechssler J, Riedel F (2001) Evolutionary dynamics on infinite strategy spaces. Econ. Theory 17:141-162.

[24] Oechssler J, Riedel F (2002) On the dynamic foundation of evolutionary stability in continuous models. J. Econ. Theory 107:223-252.

[25] Oyama D, Sandholm WH, Tercieux O (2015) Sampling best response dynamics and deterministic equilibrium selection. Theor. Econ. 10:243-281.

[26] Perkins S, Leslie D (2014) Stochastic fictitious play with continuous action sets. J. Econ. Theory 152:179-213. 
[27] Pigou AC (1920) The Economics of Welfare (London: Macmillan).

[28] Phelps S, McBurney P, Parsons S (2010) Evolutionary mechanism design: a review. Auton Agent Multi-Agent Syst. 21:237-264.

[29] Rothkopf MH, Teisberg TJ, Kahn EP (1990) Why are Vickrey auctions rare? J. Political Econ. 98:94-109.

[30] Rothkopf MH (2007) Thirteen reasons why the Vickrey-Clarke-Groves process is not practical. Oper. Res. 55:191-197.

[31] Sandholm WH (2001) Potential games with continuous player sets. J. Econ. Theory 97:81-108.

[32] Sandholm WH (2002) Evolutionary Implementation and Congestion Pricing. Rev. Econ. Stud 69:667-689.

[33] Sandholm WH (2005) Negative Externalities and Evolutionary Implementation. Rev. Econ. Stud 72:885-915.

[34] Sandholm WH (2007) Pigouvian Pricing and Stochastic Evolutionary Implementation. J. Econ. Theory 132:367-382.

[35] Sandholm WH (2010) Population Games and Evolutionary Dynamics. MIT Press, Cambridge, MA.

[36] Vickrey W (1961) Counterspeculation, auctions, and competitive sealed tenders. J. Finance 16:8-37.

[37] Zeidler E (1986) Nonlinear Functional Analysis and Its Applications, vol. I (Springer Verlag, New York). 